\title{
The Seyfert Population in the Local Universe ${ }^{1}$
}

\author{
Marcio A. G. Maia and Rodolfo S. Machado \\ GEA - Observatório do Valongo - UFRJ and Observatório Nacional/MCT, Rio de Janeiro - RJ, \\ Brazil \\ maia@ov.ufrj.br, rodolfo@on.br \\ and \\ Christopher N. A. Willmer ${ }^{2}$ \\ UCO/Lick Observatory, University of California, Santa Cruz, 95064 \\ cnaw@ucolick.org
}

\begin{abstract}
The magnitude-limited catalog of the Southern Sky Redshift Survey (SSRS2), is used to characterize the properties of galaxies hosting Active Galactic Nuclei. Using emissionline ratios, we identify a total of $162(3 \%)$ Seyfert galaxies out of the parent sample with 5399 galaxies. The sample contains 121 Seyfert 2 galaxies and 41 Seyfert 1 . The SSRS2 Seyfert galaxies are predominantly in spirals of types $\mathrm{Sb}$ and earlier, or in galaxies with perturbed appearance as the result of strong interactions or mergers. Seyfert galaxies in this sample are twice as common in barred hosts than the non-Seyferts. By assigning galaxies to groups using a percolation algorithm we find that the Seyfert galaxies in the SSRS2 are more likely to be found in binary systems, when compared to galaxies in the SSRS2 parent sample. However, there is no statistically significant difference between the Seyfert and SSRS2 parent sample when systems with more than 2 galaxies are considered. The analysis of the present sample suggests that there is a stronger correlation between the presence of the AGN phenomenon with internal properties of galaxies (morphology, presence of bar, luminosity) than with environmental effects (local galaxy density, group velocity dispersion, nearest neighbor distance).
\end{abstract}

Subject headings: galaxies: Seyfert — galaxies: AGNs — galaxies: Environmental effects

\footnotetext{
${ }^{1}$ Partly based on observations at European Southern Observatory (ESO), under the ESO-ON agreement to operate the $1.52 \mathrm{~m}$ telescope.

${ }^{2}$ on leave from Observatório Nacional/MCT, Rio de Janeiro - RJ, Brazil
} 


\section{Introduction}

Since bright AGNs are such a rare phenomenon, the way a sample is selected can affect the interpretation of the observational results. As an example, the largely accepted unified model for AGNs is sometimes challenged by the results of some analyses that may be dependent on the sample selection (e.g., Laurikainen \& Salo 1995). Seyferts selected on the basis of X-ray or UV excess are biased in favor of the type 1. On the other hand, samples selected in the IR are prone to contain galaxies undergoing strong star formation activity, and also biased in favor of luminous Seyfert nuclei. The FIR correlates weakly with the AGN emission and is very likely related to a concurrent starburst. The identification of large numbers of galaxies hosting Active Galactic Nuclei has been possible thanks to the use of spectroscopic surveys using objective prisms (e.g., Salzer et al. (2000),KISS; Gronwall, Sarajedini \& Salzer (2001) and references therein). However, since these surveys are specifically designed to detect galaxies with emission-lines, using their catalogues to compare the properties of AGN host galaxies with the general field galaxies is not straightforward. The need of an isotropic property to assure a fair selection is therefore very important.

The use of magnitude-limited surveys is an effective means of selecting galaxies in a relatively unbiased way, such that detailed comparisons between the properties of AGNs and the entire sample can be done. The first analysis of this kind was by Huchra \& Burg (1992), who used the CfA1 spectroscopic database to identify AGN hosts, and calculated their luminosity function to determine their density relative to the entire sample of galaxies. Huchra \& Burg (1992) found that AGNs dominate the bright end of the CfA1 survey luminosity function. A similar conclusion was reached by Köhler et al. (1997) who used the Hamburg-ESO prism survey, and found that the spatial densities of Seyfert 1 galaxies at $z<0.07$ are consistent with those of Huchra \& Burg (1992). A detailed analysis using a smaller sample than the CfA1 was done by Ho et al. (1997b) and Ulvestad \& Ho (2001) who selected galaxies brighter than $B_{T}=12.5$, for which high resolution (and high signal-to-noise) spectroscopy was obtained. Ho et al. (1997b) and Ulvestad \& Ho (2001) showed that $\sim 40 \%$ of all galaxies present AGN-like spectra, about $2 / 3$ of the sample being LINERS, while a proportion of $50 \%$ to $75 \%$ of all AGN are found in bulge-dominated galaxies.

The largest sample of AGNs to date is those compiled by Hao \& Strauss (2003) and Kauffmann et al. (2003), both works using the Sloan Digital Sky Survey to detect AGNs. Using the H $\alpha$ flux as an estimator of the energy output from the AGN, Hao \& Strauss (2003) find that the ratio between Seyfert 1 and Seyfert 2 galaxies is roughly the same at low luminosities, while at higher luminosities, Seyfert 1 galaxies dominate by a large margin. In that work they find that there is no correlation between the nuclear luminosity and the host galaxy luminosity.

In the present work we examine the properties of AGN hosts relative to the population of field galaxies. For this we use a sample of AGNs identified in the SSRS2 survey (da Costa et al. 1998). This survey reaches about 1 magnitude deeper than the CfA1 and contains more than twice the number of galaxies. In this work, the SSRS2 is used as a "control sample" against which several properties of the AGN hosts are compared, therefore allowing a systematic characterization of the 
properties of galaxies relative to the general field galaxy sample. This paper is divided as follows: in Section 2 we describe the sample of galaxies, followed in Section 3 several analyses of the properties of AGN hosts as a function of the internal properties of galaxies. In Section 4 we examine how the AGN phenomenon correlates with the environment where the host galaxy is located. A summary of our results is presented in Section 5 .

In the present paper we use the following cosmological parameters: $H_{0}=70 \mathrm{kms}^{-1} \mathrm{Mpc}^{-1}$, $\Omega_{m}=0.3, \Lambda=0.7$

\section{The Sample}

To characterize the AGN properties in the nearby Universe, we used an updated version of the Southern Sky Redshift Survey (SSRS2, da Costa et al. 1998). This catalog contains 5399 galaxies with $m_{B} \leq 15.5$ covering 1.69 sr of the southern sky, limited in the southern galactic hemisphere by $-40^{\circ} \leq \delta \leq-2.5^{\circ}$ and $b \leq-40^{\circ}$, and in the northern galactic hemisphere by $\delta \leq 0^{\circ}$ and $b \geq+35^{\circ}$. This updated version of SSRS2 contains more precise positional information, new or improved radial velocities, revised morphological classifications, and has had removed galaxies with magnitudes above the nominal limit as well as false detections in the HST Guide Star Catalogue. It also contains galaxies that where not included previously because of misclassification. This catalog is currently $99.99 \%$ complete in redshift, and in our database we have optical spectra available for $68 \%$ of the SSRS2 galaxies, while the remaining radial velocities were obtained from the literature.

The spectra were inspected and AGNs identified using the diagnostic diagrams of line intensity ratios proposed by Baldwin et al. (1981) and Veilleux \& Osterbrock (1987). We classify as Seyfert galaxies objects that satisfy:

$$
[N I I] \lambda 6584 / H \alpha>0.6
$$

and

$$
[O I I I] \lambda 5007 / H \beta>3
$$

In dubious cases, we also used the following ratios whenever available:

$$
[O I I] \lambda 3727 /[O I I I] \lambda 5007<1
$$

and/or

$$
[O I] \lambda 6300 / H \alpha<0.3
$$

We classify as Seyfert 1 those galaxies with broad $H \alpha$ lines in their spectra. Using the prescriptions described above, we found 98 Seyfert galaxies. Some of them were detected in the SSRS2 survey and were reported in the papers by Maia et al. (1987) and Maia et al. (1996). In cases where the Seyfert nature was dubious due to low signal to noise or small wavelength coverage of the spectra, new spectroscopic data were obtained. 
Additional Seyfert galaxies in the region covered by the SSRS2 were included after searching the NED database and the literature. The literature sometimes presents conflicting classifications, or does not state clearly how galaxies were classified. A very common confusion is to classify LINERS as Seyferts. For all candidate objects recovered from the literature, we obtained spectra, and measured the line ratios so their Seyfert nature could be confirmed. This ensures that the present catalog has a homogeneous classification procedure. A total of 64 additional galaxies were added to our list.

The catalog of SSRS2 Seyfert galaxies (hereafter, AGNC) is presented in Table 1, which contains the object name, equatorial coordinates, apparent magnitude, morphological type, axial ratio $b / a$, the heliocentric radial velocity and the Seyfert type (1 or 2).

The apparent magnitudes in Table 1 were derived from the HST Guide Star Catalogue (Lasker et al. 1990), and refer to the galaxy as a whole, there being no decomposition between the contribution from the host galaxy and the AGN. The tabulated magnitudes are shown by Alonso et al. (1994) to be isophotal magnitudes with a limiting isophote close to $26 \mathrm{mag} \mathrm{arc} \mathrm{sec}^{-2}$; these magnitudes are also very close to the $B(0)$-Zwicky magnitude system used in the CfA1 survey. The conversion between $B(0)$-Zwicky and total magnitudes is given by $B(0)$-Zwicky $=B_{T}+0.26$ (Felten 1985).

In the subsequent analyses, the observed magnitudes are corrected for galactic extinction using the DIRBE/IRAS maps (Schlegel et al. 1998), and using $A / E(B-V)=4.035$, where we assume that the bandpass of the plates used for the GSC is the same as that used for the APM survey (Maddox et al. 1990). In the analyses, the radial velocities of galaxies are corrected to the Local Group Barycenter following Yahil et al. (1977).

The absolute magnitudes are calculated using the expressions presented by Hogg (2000). For the K-corrections we use morphological-type dependent expressions due to Pence (1976).

In Figure 1 we show the distribution of apparent magnitudes of galaxies in the AGNC and the SSRS2. This figure suggests there might be a lack of AGNs for magnitude bins fainter than $\mathrm{m}_{B}=15.0$. This possible incompleteness is also suggested by the $V / V_{\max }$ statistic (Schmidt 1968), which for the SSRS2 sample is 0.47 , while for the AGNs, it is 0.38 .

An independent assessment of the catalogue completeness can be made using the completeness test $\left(T_{c}\right)$ proposed by Rauzy (2001), shown in Figure 2. In this test, the completeness is estimated by calculating for each galaxy $i$ the ratio between the number of galaxies with absolute magnitudes $M \leq M_{i}\left(r_{i}\right)$ and the number of galaxies $\left(n_{i}\right)$ visible to survey limit $\left(m_{\text {lim }}\right)$ in both cases within the volume out to $z=z_{i}$. From these one calculates the estimator

$$
\bar{\zeta}=\frac{r_{i}}{n_{i}+1}
$$

which is related to the ratio between the cumulative luminosity function

$$
\zeta=\frac{F\left(M \leq M_{i}\right)}{F\left(M \leq M_{l i m}(z)\right)}
$$


The expectation value and variance of $\zeta$ are respectively :

$$
E_{i}=\frac{1}{2}
$$

and

$$
V_{i}=\frac{1}{12} \frac{n_{i}-1}{n_{i}+1}
$$

$T_{c}$ is then defined as the ratio between the fluctuations of the cumulative luminosity function and its variance:

$$
T_{c}=\frac{\sum_{i=1}^{N_{\text {gal }}\left(\bar{\zeta}-\frac{1}{2}\right)}}{\left\{\sum_{i=1}^{N_{\text {gal }}} V_{i}\right\}^{\frac{1}{2}}}
$$

(see Rauzy (2001) for the full derivation). The underlying assumption that is made in this test is that there is no dependence between an object's luminosity and its location. This test shows that the sample completeness cannot be rejected at a confidence level greater than $95 \%$, suggesting that the incompleteness of the present sample may not be severe at the adopted limiting magnitude $\left(m_{B}=15.5\right)$.

In Figure 3 we show the radial velocity distribution of AGNC and SSRS2 galaxies. For radial velocities $\geq 13,000 \mathrm{~km} \mathrm{~s}^{-1}$ we see that both samples present few galaxies, this velocity range being populated by galaxies about 1.5 magnitudes brighter than $M^{*}$. The Kolmogorov-Smirnov test (KS), applied to the SSRS2 and AGNC samples shows that their velocity distributions differ at the $80 \%$ confidence level. This may be reflecting the fact that bright AGNs will contribute significantly to the total emission of galaxies, therefore enhancing the Malmquist bias, such that brighter and more distant galaxies will be included in the sample. The average distance, $\langle d\rangle$, of the galaxies in the samples are $\left\langle d_{S S R S 2}>=108 \mathrm{Mpc}\right.$ and $\left\langle d_{A G N C}>=100 \mathrm{Mpc}\right.$.

We show in Figure 4 the fraction of available spectra as a function of galaxy morphology for galaxies in the SSRS2, where the galaxy morphologies are coded following de Vaucouleurs et al. (1976). The SSRS2, in general, samples spectroscopically all morphologies at a similar rate, with exception of $\mathrm{T}=-1$ (S0a) and very late types, such as Sds and Irregular galaxies. This figure can also be used to estimate the fraction of AGNs that may have been missed in the SSRS2 because no spectra are available. By considering the fraction of galaxies with spectra in the SSRS2 (68\%) database, and the fraction of AGNs detected in each morphological type, the expected number of active galaxies in the $32 \%$ without previous spectra in our database should be of about 42 . This is less than the 64 galaxies recovered from the inspection of the literature and may be an indication that the AGNC is not drastically affected by the lack of spectra for $32 \%$ of SSRS2 galaxies.

\section{Properties of AGN host galaxies}

In this section we use the AGNC to characterize the properties of Seyfert galaxies, and compare them to a representative ensemble of galaxies of the Local Universe 
The total number of Seyfert galaxies identified in the SSRS2 is 162. This comprises about 3\% of the total number of galaxies in the sample (5399). This number is consistent with the proportion of $2 \%$ found by Huchra \& Burg (1992) for the 48 Seyferts of the CfA1, the $4.7 \%$ found by Hao $\&$ Strauss $(2003)$ for the SDSS and the $\sim 4 \%$ found for KISS by Gronwall, Sarajedini \& Salzer (2001). We should note that Maiolino \& Rieke (1995) find that the Revised Shapley-Ames Catalog of Bright Galaxies (RSA Sandage \& Tammann 1981), contains 5\% (91 galaxies) of AGNs, although they claim, using completeness arguments, that AGNs may comprise up to $16 \%$ of the sample. This is supported also by Ho et al. (1997a) who find that about $11 \%$ of the galaxies in a subsample of 486 nearby galaxies selected from the RSA catalog are Seyferts. In a mid-IR selected sample of 891 galaxies, Hunt \& Malkan (1999) find a fraction of 9\% of AGNs (34 type 1 and 44 type 2).

Part of these differences may be explained by the criteria used in different works to classify a galaxy as an AGN. In particular, IRAS samples are strongly biased towards luminous, dusty, early-type spiral galaxies. We should note that the present sample of AGNs identified from the SSRS2 may be deficient in low-luminosity AGNs, particularly when compared to works like Ho et al. (1997a) who used a different strategy that allowed uncovering lower luminosity AGNs, through the use of high-dispersion spectroscopy (Ho et al. 1997a).

In the AGNC catalogue, which contains 162 galaxies, there are 121 Seyfert type 2 galaxies and 41 type 1 , corresponding to a ratio of $\sim 3: 1$. For the KISS, the proportion is $\sim 2: 1$ (Gronwall, Sarajedini \& Salzer 2001), while the ratio between narrow-line and broad-line AGNs in the SDSS is also 2:1 (Hao \& Strauss 2003). Other authors that measured this proportion found ratios of 4:1 for the RSA (Maiolino \& Rieke 1995), 1:1 for the CfA1 (Huchra \& Burg 1992), and 1:1 found by Rush et al. (1993) for their $12 \mu \mathrm{m}$ flux-limited sample of galaxies.

\subsection{Morphology}

We display the fraction of galaxies as a function of morphological type in Figure 5 . The AGNC (solid line) and SSRS2 (dashed line) distributions differ at a confidence level of 99.99\%. The Seyferts are distributed preferentially among early-type spirals, confirming previous results (e.g., Moles et al. 1995; Hunt \& Malkan 1999; Gronwall, Sarajedini \& Salzer 2001), with $70 \%$ of the AGNs between the morphological types S0a and Sb, there being $\sim 5 \%$ of AGNs in ellipticals and very few hosted by spirals later than Sb. Similar results are found by Kauffmann et al. (2003) in their analysis of SDSS data. Although SDSS has no morphologies, Kauffmann et al. (2003) were able to show that most of the AGN hosts in their data are found in centrally concentrated galaxies, similar to early type galaxies. There is a high proportion of Seyferts in galaxies with type 15, which contain about $10 \%$ of the total sample of AGNs. This morphological type is used in the SSRS2 to denote galaxies presenting evidence of advanced merger event.

Evidence of segregation in terms of morphologies was presented by Hunt \& Malkan (1999), who claimed that Seyfert 1s are more often found in earlier spirals compared to Seyfert 2s. By 
analysing the KISS galaxies Gronwall, Sarajedini \& Salzer (2001) find that Seyfert 2 (and LINERS) are preferentially found in redder hosts $(B-V=0.92)$, while Seyfert 1 hosts typically have bluer colors $(B-V=0.70)$. This apparent contradiction between the two works can be explained by the use of integrated colors of galaxies i.e., the contribution of the AGN is very hard to be measured separately, added to the fact that the correlation between galaxy morphology and color has a large dispersion. Yet both works suggest that the Seyfert types are preferentially found in different types of host. The AGNC was used to investigate whether this segregation in morphologies between the Seyfert 1 and 2 hosts is found in the SSRS2 (Figure 6). By using a KS test we find that both samples may be considered identical at the $84 \%$ confidence level, showing that for the SSRS2 there is no strong correlation between the host galaxy morphology and the Seyfert type.

\subsection{Luminosities}

The distribution of absolute magnitudes, $M_{B}$ for the AGNC and SSRS2 catalogs is displayed in Figure 7 panel (a), and between the Seyfert types 1 and 2 in panel (b). Panel (a) clearly shows that galaxies hosting AGNs have a peaked distribution, while the non-actives present a tail at lower luminosities. The mean values of $M_{B}$ are: $-20.06 \pm 1.32$ for the SSRS2, $-20.59 \pm 0.87$ for AGNs, $-20.85 \pm 0.77$ for type 1 and $-20.50 \pm 0.89$ for type 2 . The evidence here is that AGNs reside preferentially in high luminosity hosts, as already suggested by Huchra \& Sargent (1973) and confirmed by Huchra \& Burg (1992), and Gronwall, Sarajedini \& Salzer (2001). Since we cannot isolate the light from the AGN from that of the host with the present data, it is unclear how much the observed distribution is due to the host galaxy and how much is due to the AGN itself. As shown by Londish et al. (2000), the correlation between the total and typical nuclear luminosities of galaxies with AGNs as measured from an HST-selected sample, is fairly tight for high-luminosity AGNs ( $\mathrm{rms} \sim 1 \mathrm{mag}$ ), but shows a broad dispersion once lower-luminosity AGNs are considered (rms $>1 \mathrm{mag}$ ). This may be explained by the difficulty in isolating the contribution from the AGN even in the case of HST data. This interpretation is supported by Hao \& Strauss (2003) who show using SDSS data, that there is no correlation between the AGN luminosity and that of the host galaxy. Notwithstanding, the tendency we have found may be attributed, at least in part, to the relation between the black hole mass and that of the galaxy bulge.

\subsection{Axial Ratios}

Since Seyfert hosts are frequently found in spiral galaxies, it is possible that optically-selected samples may be biased against identifying AGNS in edge-on systems by projection effects. This is because the gas which is ionized by the nucleus is not detected along the line of sight, being obscured by the intervening gas and dust in the plane of the galaxy. The distribution of the axial ratio $b / a$ for the non-interacting spirals of the AGNC is similar to that of a magnitude-limited

sample of thin circular disks (Maiolino \& Rieke 1995). The frequency distribution of axial ratios 
for all Seyferts is presented in Figure 8 panel (a). The lack of objects with very small axial ratios $b / a$ is expected because of the disk thickness and also to the less numerous edge-on systems in magnitude-limited samples. The small decrease in frequencies for $b / a$ above 0.8 may be attributed to the existence of noncircular disks.

In panel (b) of Figure 8 we show the distribution of axial ratios for Seyfert 2 galaxies (solid line) and Seyfert 1 (dashed line). Seyfert 2 s tend to be more abundant at smaller values of $b / a$ while Seyfert 1s are commoner at $b / a$ closer to 1 . A KS test shows that both samples differ at the $97 \%$ confidence level. This behavior is consistent with the interpretation that part of Seyfert 2 galaxies are Seyfert 1s heavily obscured in more inclined systems.

\subsection{Presence of Bar}

Bars are claimed to be detected in up to $50 \%$ of normal galaxies (e.g., Selwood \& Wilkinson 1993; Knapen et al. 2000), although some authors claim that the fraction may be as high as $\approx 70 \%$ (e.g., Hunt \& Malkan 1999). The presence of this non-axisymmetric gravitational perturbation can induce radial gas inflow, fueling the Seyfert activity as described by Heller \& Shlosman (1994). The incidence of bars as a function of morphological type was reported by Ho et al. (1997b), although Knapen et al. (2000) do not find any trend. Laine et al. (2002) using IR HST data, find that bars are more abundant in Seyfert hosts (73\%) than in non-Seyferts (50\%). On the other hand, Moles et al. (1995), Mulchaey \& Regan (1997) and Malkan et al. (1998) find similar occurrence of barred systems in Seyferts and normal galaxies.

In order to detect the presence of bars, DSS images of SSRS2 galaxies were visually inspected (by MAGM). We do not differentiate between strong and weak bars. We are not able to identify the totality of bars due to the saturation of the central parts of galaxies in DSS plates or the inability to detect bars in edge-on systems and for galaxies with small apparent sizes. Nevertheless, we are able to detect, at least, the relative differences between the SSRS2 and AGNC, since the procedure used to identify bars for both samples was identical.

The fraction of barred galaxies as a function of morphological type, for the SSRS2 and the AGNC, is displayed in Figure 9. We find that the SSRS2 shows no dependence between the fraction of galaxies with bars and morphology. On the other hand, the higher frequency of barred galaxies in the AGNC sample is noticeable. The barred fraction in the SSRS2 is 14.1\% while for the AGNC is $28.4 \%$. These distributions are different at the $99.97 \%$ confidence level. If we consider only the interval of morphological types between S0a and Sbc, where we have the majority of Seyferts, the result is still the same. The percentage of barred systems in the SSRS2 is smaller than that found in the RSA catalog by Ho et al. (1997b). This discrepancy can be partly explained by our inability to detect bars visually in galaxies with apparent diameters smaller than $0.5^{\prime}$.

The distribution of the fraction of galaxies with bars as a function of morphology, but now discriminating between Seyfert 1 and Seyfert 2 galaxies is shown in Figure 10. The distribution 
is fairly noisy, and by applying a KS test we find that the difference between both samples is not significant.

\subsection{The Spatial Density of Seyferts}

To determine the spatial density of Seyfert galaxies in the SSRS2, we use the the nonparametric step-wise maximum likelihood method of Efstathiou et al. (1988) to measure the luminosity function of AGN hosts. Although in principle we could calculate the parametric Schechter (1976) function, we have chosen not to do so because of the small sample size and the strong correlation between the different Schechter parameters. By calculating the step-wise maximum likelihood for both the SSRS2 sample and the Seyfert galaxy subsample we can compare the spatial density of AGN hosts relative to the parent sample as a function of absolute magnitude.

Prior to the calculation of the luminosity function, galaxies with corrected radial velocities smaller than $500 \mathrm{kms}^{-1}$ or greater than $15000 \mathrm{kms}^{-1}$ are removed from the sample. The SWML is calculated for galaxies in the range $-22.5 \leq M_{B} \leq-16.0$, and is shown in Figure 11 where the AGN sample is represented as solid squares. The error bars were obtained following Efstathiou et al. (1988). The histogram showing the number of galaxies per absolute magnitude bin is shown in the lower part of the figure.

In Figure 11, in addition to the AGNC non-parametric luminosity function, we show previous determinations by other authors converted into the cosmological model and magnitude system adopted in this paper: Huchra \& Burg (1992) represented as crosses; Köhler et al. (1997), open circles; Londish et al. (2000), open triangles; Ulvestad \& Ho (2001), shown as solid triangles, and Gronwall, Sarajedini \& Salzer (2001) as stars. The figure also shows the SWML calculated for the SSRS2 parent sample (solid triangles). The AGNC luminosity function is in very good agreement with Gronwall, Sarajedini \& Salzer (2001) and Huchra \& Burg (1992). The agreement with both Köhler et al. (1997) and Londish et al. (2000) is not as good, which could be explained by the rather smaller samples of those two works. The AGNC number density (as well as those of Huchra \& Burg 1992; Gronwall, Sarajedini \& Salzer 2001) are systematically lower than measured by Ulvestad \& Ho (2001), probably reflecting the presence of LINERs in the AGN sample of the latter work.

In Figure 12 we show the ratio between the number density of AGN hosts relative to the that of the parent sample as a function of absolute magnitude. In the figure we show this ratio as a differential distribution (open triangles) where we calculate the ratio at each magnitude bin. We also show the cumulative distribution (solid circles), where all galaxies in each subsample in a given magnitude bin and brighter are added. The figure, also suggests that on average slightly more that $1 \%$ of galaxies down to $M_{B}=-16$ are AGN hosts. However, as brighter absolute magnitudes are probed, the proportion of AGN hosts increases, a trend already noted by Huchra \& Sargent (1973) and Huchra \& Burg (1992). At $M^{*}$, corresponding to $M_{B} \sim-20.5$, AGNs are found in $\sim 3 \%$ of galaxies. 


\section{Dependence of the AGN Phenomenon on the Environment}

In this section, we examine how the AGN phenomenon correlates with the environment where the host galaxy is located. An unresolved issue in the study of AGNs is fueling the "central motor" of the AGN, whereby mass in the form of gas (or stars) is fed into the mass concentration generating the AGN. Among the mechanisms proposed to explain the transportation of gas from the disk of a spiral galaxy to its nucleus, is the interaction with a close galaxy (e.g.; Noguchi 1988; Barnes \& Hernquist 1992). Several observational studies claim that AGN hosts show an excess of companions relative to normal galaxies (e.g.; Stauffer 1982; Dahari 1985; MacKenty 1990; Laurikainen et al. 1994; Rafanelli et al. 1995), while other works find no evidence of such an excess of companions (e.g., Ulvestad \& Wilson 1985; Fuentes-Williams \& Stocke 1988; de Robertis et al. 1998). Part of this controversy may be explained by the way the AGN and control samples are generated. The influence of the environment over AGN phenomenon can be examined from scales ranging from the typical separation of close companions $(\sim 100 \mathrm{kpc})$ to that of groups and clusters $(\sim 1 \mathrm{Mpc})$. In particular, at large scales, it is possible that even competing mechanisms could play a complex role. In such places the AGN may be fueled from gas captured from close companion, during an interaction with a close companion. On the other hand, the central monster could be "starved" by the removal of gas from the galaxy through the mechanism of ram pressure stripping of the intracluster medium, as has already been proposed to explain anemic galaxies.

\subsection{Seyferts and Groups}

One of the first works showing that Seyfert galaxies follow the general trend of clustering in the same way as normal galaxies do, but avoiding rich clusters, was by Petrosian (1982). This trend was subsequently confirmed by Dressler et al. (1985), who found that the fraction of AGNs in rich clusters is $\approx 1 \%$, similar to the proportion of Seyfert galaxies in the field galaxy population. In a sample of galaxies selected in regions of high density of galaxies, typical of the central parts of clusters and compact groups of galaxies, Maia et al. (1994) find an excess of Seyferts when compared to a control sample of galaxies from regions with very low density of objects. This result is confirmed by Focardi et al. (2002), using the UZC-CG catalogue to identify compact groups of galaxies. The proportion of galaxies hosting an AGN in compact groups is still not well known, and estimates range from $70 \%$ (Coziol et al. 2000) to 50\% (Shimada et al. 2000). In fact, the latter authors conclude, that after correcting for the different morphological distributions of those environments, the dense galaxy environment in the compact groups triggers neither the AGN activity nor the nuclear starburst.

The proportion of AGNs in lower density ("loose") groups is even less well known. Kollastchny \& Friecke (1989) compare the emission-line properties of galaxies in a small sample of groups with

and without Seyferts. They conclude that groups containing Seyferts contain more frequently objects with strong emission lines indicative of intense star formation, relative to groups without 
Seyfert galaxies. In another analysis of loose groups of galaxies, Kelm et al. (1998) find no significant differences in the dynamical properties (velocity dispersions) of groups harboring Seyferts as compared to those without Seyferts.

We re-examine the correlation between the presence of the AGN phenomenon with local density by identifying groups in the SSRS2. This is done using a percolation algorithm applied to the SSRS2 catalog. We generated a catalog of groups of galaxies similarly to Merchán et al. (2000), for which we examined the distribution of Seyfert galaxies. The algorithm is that described by Huchra \& Geller (1982) and Maia et al. (1989). Since we are interested in "real physical systems", the adopted friends-of-friends algorithm, searches for possible group member galaxies, keeping a fixed surrounding density contrast $(\delta \rho / \rho)$ relative to the mean density of galaxies of the entire sample. Groups were selected so they correspond to a density enhancement $\delta \rho / \rho=80$. This level was adopted following Ramella et al. (1997) who showed that this density contrast level gives the best compromise between identifying as many physical loose groups as possible, including systems with high velocity dispersion, but minimizing contamination of the catalog by pseudo-groups as well as groups with interlopers. The search for companions around galaxies is carried out taking into account projected separations satisfying

$$
D_{12}=2 \sin \left(\frac{\theta_{12}}{2}\right) \frac{V}{H_{0}} \leq D_{L}
$$

and with line-of-sight velocity differences,

$$
V_{12}=\left|V_{1}-V_{2}\right| \leq V_{L}
$$

In the above expressions $V=\left(V_{1}+V_{2}\right) / 2, V_{1}$ and $V_{2}$ are the radial velocities of the galaxies, and $\theta_{12}$ their angular separation. The quantities $D_{L}$ and $V_{L}$ are search parameters scaled according to the expressions below in order to take into account the variation in the sampling of the galaxy luminosity function, $\phi(M)$, with distance

$$
D_{L}=D_{0} R \quad ; \quad V_{L}=V_{0} R
$$

where

$$
\begin{gathered}
R=\left[\int_{-\infty}^{M_{12}} \Phi(M) d M / \int_{-\infty}^{M_{l i m}} \Phi(M) d M\right]^{-1 / 3} \\
M_{l i m}=m_{l i m}-25-5 \log \left(V_{f} / H_{0}\right) \\
M_{12}=m_{l i m}-25-5 \log \left[\left(V_{1}+V_{2}\right) / 2 H_{0}\right]
\end{gathered}
$$

$D_{0}$ is the selection parameter at a fixed fiducial radial velocity, $V_{f}$. $V_{L}$ is scaled in the same way as $D_{L}$. The adopted values for $D_{0}$ and $V_{0}$ are $0.352 \mathrm{~h}^{-1} \mathrm{Mpc}$ and $350 \mathrm{~km} \mathrm{~s}^{-1}$ respectively. The apparent magnitude, $m_{\text {lim }}=m_{B}=15.5$, while the luminosity function parameters are $\phi^{*}=0.0137$ galaxies $\mathrm{mag}^{-1} \mathrm{Mpc}^{-3}, M_{B}^{*}=-19.40$ and $\alpha=-1.08$ (Marzke et al. 1998).

The resulting group catalog contains systems with at least 4 members and mean radial velocities $V_{g} \leq 12,000 \mathrm{~km} \mathrm{~s}^{-1}$ (see Merchán et al. (2000) for more details). The algorithm also outputs lists 
of galaxies for which no companions are found ("isolated"), as well as binary and triple systems. The distribution of AGNC and the entire SSRS2 in each of these multiplicity classes is presented in Table 2. An inspection of the table suggests that AGNs tend to be more common in binary systems, showing no preference for being located in groups with three or more members when compared to the control sample.

The catalogue of groups was also used to examine the distributions of velocity dispersions for groups containing Seyferts against the entire group sample. If galaxy interactions are supposed to enhance/trigger the nuclear activity, we should expect that the systems presenting the lowest velocity dispersions are more likely to contain Seyfert hosts. The median values are $166 \mathrm{kms}^{-1}$ for groups with Seyferts and $170 \mathrm{~km} \mathrm{~s}^{-1}$ for the entire sample, which are essentially identical. The same conclusion is found by applying the KS-test to the distribution of velocity dispersions, therefore confirming the results of Kelm et al. (1998). For binaries we find a very weak tendency (at the $50 \%$ confidence level) for Seyfert galaxies to be present in systems with higher median velocity dispersions $\left(102 \mathrm{~km} \mathrm{~s}^{-1}\right)$, relative to the entire sample $\left(78 \mathrm{~km} \mathrm{~s}^{-1}\right)$.

The average group density, $\rho_{g}$, can be calculated by means of the expression $\rho_{g}=3 N_{g} / 4 \pi r^{3}$ where, $N_{g}$ is the number of galaxies and $r$ the mean pairwise separation of a given group. The distributions of $\rho_{g}$, show no significant difference between Seyfert and non-Seyfert groups for binary, triple or even higher multiplicity orders. In Figure 13 we display the median values and upper and lower quartiles for $\rho_{g}$ for several group multiplicities, and there is no evidence of the AGN phenomenon being correlated with the group density.

\subsection{The Nearest Neighbor Distance and Local Density}

One of the suggestions of MacKenty (1990) is that close companion galaxies enhance the star formation rather than directly enhancing Seyfert-like activity. On the other hand Laurikainen \& Salo (1995) conclude that Seyferts have on average about twice the number of companions than other galaxies in their control sample. Dultzin-Hacyan et al. (1999) also find a significant excess of large companions for Seyfert 2 galaxies but no such evidence for Seyfert 1. On the other hand, Rafanelli et al. (1995) report no difference between the presence of close companions for Seyfert galaxies relative to non-active galaxies. We address this issue estimating the separation of the nearest neighbor, $S$, for galaxies in our sample.

In this analysis we use the SSRS2 catalogue restricting it to contain objects with velocities smaller than $12000 \mathrm{~km} \mathrm{~s}^{-1}$, therefore minimizing the problem of decreasing completeness of the sample with distance. We also neglect galaxies which are located at projected distances $<1 \mathrm{Mpc}$ from the survey boundaries. We further restrict the sample to galaxies with morphological types between S0a and Sbc, the interval which contains approximately $80 \%$ of the Seyfert galaxy sample. This is done to minimize the bias induced by the morphology-density relation, since we have a negligible fraction of Seyferts hosted by elliptical galaxies, which are objects generally found in 
high density environments. The distributions of the nearest neighbor, $S$, for AGNC and SSRS2 samples were examined using the KS test which shows that both samples are part of the same parent population at the $98 \%$ confidence level. If we compare the distribution of the second nearest neighbor, a similar result is found. We find a trend of Seyfert 1s presenting, on average, closer companions than the type $2 \mathrm{~s}$, but the result is not statistically significant.

The nearest neighbor distance is also used to compute the maximum tidal influence, $Q$, that a companion may exert on the AGN host galaxy. This tidal influence is proportional to the companion's mass divided by the cube of the separation, $S$. Assuming that light traces mass, the tidal parameter for each host is $Q \propto L / S^{3}$. The distribution of $Q$ values for the AGNC and SSRS2 are identical at the $86 \%$ confidence level. The Seyfert types 1 and 2 are considered the same only at the $20 \%$ confidence level, with type 1 s presenting higher values of $Q$, compared to type 2 s.

\section{Summary}

In the present work we have used the magnitude-limited SSRS2 to identify galaxies with active galactic nuclei, and to investigate how the properties of AGN host galaxies relate to general population of galaxies in the parent sample. The main results of this work are summarized below.

- We find that 162/5339 (3\%) of the galaxies in the SSRS2 are Seyfert galaxies. This number is comparable to other works studying the local universe (e.g., Huchra \& Burg 1992; Ho 2001; Gronwall, Sarajedini \& Salzer 2001; Hao \& Strauss 2003), as well as with the number of AGNs detected in a high redshift sample (e.g., Sarajedini 2002).

- The ratio of Seyfert $2 \mathrm{~s}$ to $1 \mathrm{~s}$ is about $3: 1$.

- The majority of the AGN host galaxies in the SSRS2 are in systems classified morphologically betwen $\mathrm{S0a}$ and $\mathrm{Sb}$, which are galaxies containing significant bulge components. This result is consistent with findings of Ho (2001). We find that $\sim 10 \%$ of the Seyfert galaxies are found in hosts with indication of an ongoing merger event. There is no significant difference between the morphological distributions of Seyfert 1 relative to Seyfert 2 hosts.

- The AGNs are preferentially detected in high luminosity hosts $\left(M<M^{*}+1\right)$. This result is similar to previous findings (e.g., Huchra \& Burg 1992), though in conflict with that found by Hao \& Strauss (2003) using the SDSS database. This discrepancy can be explained by the fact that the present sample does not allow separating the contribution to the total luminosity due to the AGN from that of the host galaxy.

- Seyfert galaxies are twice as frequent in barred hosts than in galaxies without nuclear activity.

- Using a percolation algorithm we find that Seyfert galaxies are more commonly found in binary systems than in groups with higher multiplicities or as "isolated" galaxies, when compared to galaxies in the parent sample. 
- We do not find strong correlation between the presence of an AGN with indicators of the strength of gravitational perturbation by companions, such as the projected local density, the group velocity dispersion, the nearest neighbor distance and the maximum tidal influence.

- We find marginal evidence that Seyfert 1 galaxies have closer companions and are more susceptible to tidal effects than Seyfert 2 galaxies are.

- The results above suggest that the large-scale environment does not seem to play an important role in enhancing the AGN activity. On the other hand, internal characteristics such as morphology, luminosities, existence of bars and other asymmetries are more strongly correlated with the presence of the AGN.

The authors thank the referee, Dr. John Huchra, whose suggestions allowed us to improve the text. We also thank Alberto Rodríguez-Ardilla and Lei Hao for useful discussions and Caryl Gronwall, Vicki Sarajedini and John Salzer for providing the KISS non-parametric luminosity function. This research has made use of NASA/IPAC Extragalactic Database (NED). RSM acknowledges financial support from PIBIC-CNPq scholarship, MAGM to CNPq grant 301366/86-1 and FAPERJ E-26/171.619/2001, and CNAW to NSF AST 95-29028 and AST 00-71198.

\section{REFERENCES}

Alonso, M.V., da Costa, L.N., Latham, D.W., Pellegrini, P.S., \& Milone, A.E. 1994, AJ 108, 1987

Baldwin, J.A., Phillips, M.M., \& Terlevich, R. 1981, PASP 93, 5

Barnes, J.E., \& Hernquist, L. 1992, ARA\&A 30, 705

Coziol, R., Iovino, A., \& de Carvalho, R.R. 2000, AJ 120, 47

da Costa, L.N., Willmer, C.N.A, Pellegrini, P.S., Chaves, O.L., Rite, C., Maia, M.A.G., Geller, M.J., Latham, D.W., Kurtz, M.J., Huchra, J.P., Ramella, M., Fairall, A.P., Smith, C., \& Lipari, S. 1998, AJ 116, 1

Dahari, O. 1985, AJ 90, 1772

de Robertis, M.M., Yee, H.K.C., \& Hayhoe, K. 1998, ApJ 496, 93

de Vaucouleurs, G., de Vaucouleurs, A., \& Corwin, H. 1976, Second reference Catalog of Bright Galaxies, (Austin: University of Texas Press)

Dressler, A., Thompson, I.B., \& Schectman, S.A. 1985, ApJ 288, 481

Dultzin-Hacyan, D., Krongold, Y., Fuentes-Gurudi, I., \& Marziani, P. 1999, ApJ 513, L111 
Efstathiou, G., Ellis, R.S., \& Peterson, B.A. 1988, MNRAS 232, 431

Felten, J.E. 1985, Comments Astrophys. 11, 53

Focardi, P., Kelm, B., Zitelli, V. \& Sarti, G. 2002, astro-ph/0209374

Fuentes-Williams, T., \& Stocke, J.T. 1988, AJ 96, 1235

Gronwall, C., Sarajedini, V.L., \& Salzer, J.J. 2001, in "AGN Surveys" , I.A.U. Colloquium 184, eds. R.F. Green, E.Ye. Khachikian, \& D.B. Sanders ASP Conference Series (San Francisco: ASP), astro-ph/0111519

Hao, L., \& Strauss, M.A. 2003, to appear in "Proceedings of The Carnegie Observatories Centennial Symposium I", http://astro.princeton.edu/ haol/thesis.html

Heller, C.H., \& Shlosman, I. 1994, ApJ 424, 84

Ho, L. C. 2001, in "AGN Surveys", I.A.U. Colloquium 184, ASP Conference Series (San Francisco: ASP), 12

Ho, L.C., Filipenko, A.V., \& Sargent, W.L.W 1997a, ApJ 487, 568

Ho, L.C., Filipenko, A.V., \& Sargent, W.L.W 1997b, ApJS 112, 315

Hogg, D.W. 2000, astro-ph/9905116 v4

Huchra, J.P., \& Burg, R. 1992, ApJ 393, 90

Huchra, J.P., \& Geller, M.J. 1982, ApJ 257, 423

Huchra, J.P., \& Sargent, W.L. 1973, ApJ 186, 433

Hunt, L.K., \& Malkan, M.A. 1999, ApJ 516, 660

Kauffmann, G., Heckman, T.M., Tremonti, C., Brinchmann, J., Charlot, S., White, S.D.M., Ridgway, S., Brinkmann, J., Fukugita, M., Hall, P., Ivezic, Z., Richards, G., \& Schneider, D. 2003, astro-ph/0304239

Kelm, B., Focardi, P., \& Palumbo, G.G.C. 1998, A\&A 335, 912

Knapen, J.H., Shlosman, I., and Peletier, R.F. 2000, ApJ 529, 93

Köhler, T., Groote, D., Reimers, D., \& Wisotzki, L. 1997, A\&A, 325, 502

Kollatschny, W., \& Fricke, K.J. 1989, A\&A 219, 34

Laurikainen, E. \& Salo, H. 1995, A\&A 293, 683

Laurikainen, E., Salo, H., Teerikorpi, P., \& Petrov 1994, A\&AS 108, 491 
Laine, S., Shlosman, I., Knapen, J.H. \& Peletier, R.F. 2002, ApJ 567, 97

Lasker, B.M., Sturch, C.R., McLean, B.J., Russell, J.L., Jenkner, H., \& Shara, M.M. 1990, AJ 99, 2019

Londish, D., Boyle, B. J., \& Schade, D. J. 2000, MNRAS 318, 411

MacKenty, J.W. 1990, ApJ 343, 125

Maddox, S.J., Efstathiou, G., \& Sutherland, W. J. 1990, MNRAS 246, 433

Maia, M.A.G., da Costa, L.N., \& Latham, D.W. 1989, ApJS 69, 809

Maia, M.A.G., da Costa, L.N., Willmer, C.N.A., Pellegrini, P.S., Rite, C. 1987, AJ 93, 546

Maia, M.A.G., Pastoriza, M.G., Bica, E., Dottori, H. 1994, ApJS 93, 425

Maia, M.A.G., Suzuki, J.A., da Costa, L.N., Willmer, C.N.A.; Rite, C. 1996, A\&A 117, 487

Maiolino, R., \& Rieke, G.H. 1995, ApJ 454, 95

Malkan, M.A., Gorgian, V., \& Tam, R. 1998, ApJS 117, 25

Marzke, R.O., da Costa, L.N., Pellegrini, P.S., Willmer, C.N.A., \& Geller, M.J. 1998, ApJ 503, 617

Merchán, M.E., Maia, M.A.G., Lambas, D.G. 2000, ApJ 545, 26

Moles, M., Márquez, I., \& Pérez, E. 1995, ApJ 438, 604

Mulchaey, J.S., \& Regan, M.W. 1997, ApJ 482, L135

Noguchi, M. 1988, A\&A 203, 259

Pence, W. 1976, ApJ 203, 39

Petrosian, A.R. 1982, Asrofizika 18, 548

Rafanelli, P., Violato, M., \& Baruffolo, A. 1995, AJ 109, 1546

Ramella, M., Pisani, A., \& Geller, M.J. 1997, AJ 113, 483

Rauzy, S. 2001, MNRAS 324, 51

Rush, B., Malkan, M.A., \& Spinoglio, L. 1993, ApJS 89, 1

Salzer, J.J., Gronwall, C., Lipovetsky, V. A., Kniazev, A., Moody, J.W., Boroson, T.A., Thuan, T.X, Izotov, Y.I., Herrero, J.L., and Frattare, L.M., 2000, AJ 120, 80

Sandage, A.R. \& Tammann, G.A. 1981, A Revised Shapley-Ames Catalog of Bright Galaxies (Washington: Carnegie Inst. Washington) 
Sandage, A., Tammann, G.A., \& Yahil, A. 1979, ApJ 232, 352

Sarajedini, V.L. 2002, in "AGN Surveys, ASP Conference Proceedings, Vol. 284. Edited by R.F. Green, E.Ye. Khachikian, and D.B. Sanders.", (San Francisco: Astronomical Society of the Pacific, in press)

Schechter, P. 1976, ApJ 203, 297

Schlegel, D.J., Finkbeiner, D.P., \& Davis, M. 1998, ApJ 500, 525

Schmidt, M. 1968, ApJ 151, 393

Selwood, J.A., \& Wilkinson, A. 1993, Rep. Prog. Phys. 56, 173

Shimada, M., Ohyama, Y., Nishiura, S, Muryama, T., \& Taniguchi, Y. 2000, AJ 119, 2664

Stauffer, J.R. 1982, ApJ 262, 66

Ulvestad, J.S., \& Ho, L.C. 2001, ApJ 558, 561

Ulvestad, J.S., \& Wilson, A.S. 1985, ApJ 285, 439

Veilleux, S. \& Osterbrock, D.E. 1987, ApJS 63, 295

Yahil, A., Tammann, G.A., \& Sandage, A. 1977, ApJ 217, 903

This preprint was prepared with the AAS $\mathrm{LAT}_{\mathrm{E}} \mathrm{X}$ macros v5.0. 
Table 1. SSRS2 Seyfert Galaxies

\begin{tabular}{|c|c|c|c|c|c|c|c|c|}
\hline $\begin{array}{l}\text { Coordinates } \\
\alpha\left(\begin{array}{ll}h & m s\end{array}\right)\end{array}$ & $\begin{array}{c}(\mathrm{J} 2000.0) \\
\delta\left({ }^{\circ},{ }^{\prime \prime}\right)\end{array}$ & Identification & $m_{S S R S}$ & $\begin{array}{l}\text { Velocity } \\
k m s^{-1}\end{array}$ & Type & Bar & $b / a$ & Sey \\
\hline 000332.1 & -104441 & NGC 7808 & 14.47 & 8922 & -2 & $\mathrm{~N}$ & 1.00 & S1 \\
\hline 005329.8 & -084604 & NGC 0291 & 14.64 & 5695 & 2 & $\mathrm{Y}$ & 0.45 & $\mathrm{~S} 2$ \\
\hline 001001.0 & -044238 & MRK 0937 & 14.70 & 8851 & 3 & $\mathrm{~N}$ & 1.00 & S1 \\
\hline 001054.3 & -210403 & ESO 538- G 025 & 15.02 & 7782 & 2 & $\mathrm{~N}$ & 0.22 & $\mathrm{~S} 2$ \\
\hline 001835.9 & -070256 & GSC 467000946 & 14.36 & 5620 & 0 & $\mathrm{~N}$ & 1.00 & $\mathrm{~S} 2$ \\
\hline 001944.0 & -140718 & IC 0009 & 15.42 & 12622 & 0 & $\mathrm{~N}$ & 0.75 & $\mathrm{~S} 2$ \\
\hline 002600.0 & -025507 & MCG -01-02-013 & 15.49 & 20282 & 15 & $\mathrm{~N}$ & 0.72 & $\mathrm{~S} 2$ \\
\hline 003053.8 & -09 1207 & MCG -02-02-035 & 15.28 & 5939 & -2 & $\mathrm{~N}$ & 0.50 & $\mathrm{~S} 2$ \\
\hline 003413.8 & -212621 & ESO 540- G 001 & 13.96 & 8030 & 4 & $\mathrm{Y}$ & 0.69 & $\mathrm{~S} 1$ \\
\hline 003548.8 & -133638 & NGC 0166 & 15.18 & 6020 & 0 & $\mathrm{~N}$ & 0.33 & $\mathrm{~S} 2$ \\
\hline 003818.4 & -145007 & MCG -03-02-027 & 15.40 & 10971 & 3 & $\mathrm{~N}$ & 0.67 & $\mathrm{~S} 2$ \\
\hline 004111.7 & -210754 & ESO 540- G 014 & 15.38 & 1653 & -5 & $\mathrm{~N}$ & 0.50 & $\mathrm{~S} 2$ \\
\hline 004252.8 & -233229 & NGC 0235A & 14.18 & 6589 & 15 & $\mathrm{~N}$ & 0.44 & $\mathrm{~S} 2$ \\
\hline 005454.5 & -320154 & ESO 411- G 029 & 15.04 & 9622 & 0 & $\mathrm{~N}$ & 0.38 & $\mathrm{~S} 2$ \\
\hline 005502.6 & -190018 & ESO 541- G 001 & 13.61 & 6366 & 3 & $\mathrm{Y}$ & 0.62 & $\mathrm{~S} 2$ \\
\hline 005822.2 & -363937 & ESO 351- G 025 & 15.12 & 10415 & 2 & $\mathrm{~N}$ & 0.58 & $\mathrm{~S} 2$ \\
\hline 010217.4 & -194009 & ESO 541-IG 012 NED01 & 15.22 & 16905 & 15 & $\mathrm{~N}$ & 0.85 & $\mathrm{~S} 2$ \\
\hline 010847.6 & -155034 & IC 0078 & 14.46 & 12066 & 1 & $\mathrm{~N}$ & 0.41 & $\mathrm{~S} 2$ \\
\hline 011127.5 & -380501 & NGC 0424 & 13.90 & 3496 & 0 & $\mathrm{~N}$ & 0.50 & S1 \\
\hline 011219.2 & -320343 & NGC 0427 & 14.87 & 10011 & -2 & $\mathrm{Y}$ & 0.62 & $\mathrm{~S} 1$ \\
\hline 011407.0 & -323903 & IC 1657 & 13.12 & 3564 & 3 & $\mathrm{~N}$ & 0.25 & $\mathrm{~S} 2$ \\
\hline 011925.0 & -154107 & MCG -03-04-046 & 15.26 & 15234 & 4 & $\mathrm{Y}$ & 1.00 & $\mathrm{~S} 2$ \\
\hline 012354.4 & -350356 & NGC 0526A & 14.66 & 5725 & 15 & $\mathrm{~N}$ & 0.53 & $\mathrm{~S} 2$ \\
\hline 013150.4 & -330810 & ESO 353- G 009 & 14.07 & 4970 & 2 & $\mathrm{Y}$ & 1.00 & $\mathrm{~S} 2$ \\
\hline 013924.8 & -092404 & NGC 0640 & 15.45 & 7490 & 2 & $\mathrm{~N}$ & 0.67 & $\mathrm{~S} 2$ \\
\hline 014337.6 & -334220 & ESO 353- G 038 & 14.85 & 8884 & 5 & $\mathrm{~N}$ & 0.36 & $\mathrm{~S} 2$ \\
\hline 015141.7 & -361116 & ESO 354- G 004 & 15.08 & 10045 & 3 & $\mathrm{~N}$ & 0.93 & $\mathrm{~S} 1$ \\
\hline 015951.3 & -065025 & IC 0184 & 14.87 & 5382 & 1 & $\mathrm{~N}$ & 0.50 & $\mathrm{~S} 2$ \\
\hline 020106.5 & -064856 & NGC 0788 & 13.50 & 4078 & -5 & $\mathrm{~N}$ & 0.77 & $\mathrm{~S} 2$ \\
\hline 020653.1 & -362708 & NGC 0824 & 14.05 & 5836 & 2 & $\mathrm{Y}$ & 0.95 & $\mathrm{~S} 2$ \\
\hline 020920.9 & -100800 & NGC 0833 & 13.64 & 3867 & 3 & $\mathrm{~N}$ & 0.47 & $\mathrm{~S} 2$ \\
\hline $02 \quad 1011.4$ & -090336 & GSC 528100378 & 15.23 & 12491 & 3 & $\mathrm{~N}$ & 0.50 & $\mathrm{~S} 2$ \\
\hline 022440.6 & -190830 & ESO 545- G 013 & 13.59 & 10253 & 3 & $\mathrm{~N}$ & 0.73 & $\mathrm{~S} 1$ \\
\hline 023005.5 & -085953 & MCG -02-07-024 & 15.25 & 4859 & -2 & $\mathrm{~N}$ & 0.86 & S1 \\
\hline 023151.0 & -364016 & IC 1816 & 13.66 & 5215 & 3 & $\mathrm{~N}$ & 0.93 & $\mathrm{~S} 2$ \\
\hline 023437.8 & -084715 & NGC 0985 & 15.00 & 19150 & 15 & $\mathrm{~N}$ & 1.00 & S1 \\
\hline 023513.5 & -293617 & ESO 416- G 002 & 15.06 & 17700 & 1 & $\mathrm{~N}$ & 0.50 & S2 \\
\hline
\end{tabular}


Table 1-Continued

\begin{tabular}{|c|c|c|c|c|c|c|c|c|}
\hline $\begin{array}{l}\text { Coordinates } \\
\alpha\left(\begin{array}{lll}h & m s\end{array}\right)\end{array}$ & $\begin{array}{c}(\mathrm{J} 2000.0) \\
\delta\left({ }^{\circ},{ }^{\prime \prime \prime}\right)\end{array}$ & Identification & $m_{S S R S}$ & $\begin{array}{c}\text { Velocity } \\
k m s^{-1}\end{array}$ & Type & Bar & $b / a$ & Sey \\
\hline 023845.2 & -304824 & ESO 416- G 005 & 15.34 & 18587 & 5 & $\mathrm{~N}$ & 0.80 & S1 \\
\hline 024104.8 & -081521 & NGC 1052 & 12.08 & 1519 & -3 & $\mathrm{~N}$ & 0.70 & $\mathrm{~S} 2$ \\
\hline 024138.7 & -281017 & IC 1833 & 14.28 & 4952 & -2 & $\mathrm{~N}$ & 0.58 & $\mathrm{~S} 2$ \\
\hline 024307.8 & -084626 & NGC 1071 & 15.41 & 11302 & 3 & $\mathrm{~N}$ & 0.45 & $\mathrm{~S} 2$ \\
\hline 024402.9 & -261111 & ESO 479- G 030 & 15.29 & 10504 & 2 & $\mathrm{~N}$ & 0.24 & $\mathrm{~S} 2$ \\
\hline 024619.0 & -301629 & NGC 1097 & 10.23 & 1195 & 3 & $\mathrm{~N}$ & 0.62 & S1 \\
\hline 024933.8 & -384612 & ESO 299- G 020 & 13.96 & 5008 & 1 & $\mathrm{~N}$ & 0.45 & S2 \\
\hline 025140.3 & -163904 & NGC 1125 & 13.87 & 3303 & 0 & $\mathrm{Y}$ & 0.50 & S2 \\
\hline 025609.8 & -134107 & MCG -02-08-031 & 15.38 & 6935 & 3 & $\mathrm{~N}$ & 0.50 & $\mathrm{~S} 2$ \\
\hline 025621.5 & -321106 & ESO 417- G 006 & 14.34 & 4947 & -2 & $\mathrm{~N}$ & 0.83 & $\mathrm{~S} 2$ \\
\hline 025749.6 & -101007 & MCG -02-08-033 & 14.02 & 4516 & 3 & $\mathrm{~N}$ & 0.30 & $\mathrm{~S} 2$ \\
\hline 030004.3 & -104928 & MCG -02-08-038 & 15.27 & 9770 & 0 & $\mathrm{Y}$ & 0.50 & S1 \\
\hline 030030.7 & -112407 & MCG -02-08-039 & 14.57 & 8989 & 3 & $\mathrm{Y}$ & 0.69 & $\mathrm{~S} 2$ \\
\hline 030213.2 & -233520 & GSC 643800439 & 14.93 & 10514 & -2 & $\mathrm{~N}$ & 0.39 & S1 \\
\hline 030810.8 & -225739 & NGC 1229 & 14.82 & 10676 & 1 & $\mathrm{~N}$ & 0.69 & S2 \\
\hline 031114.7 & -085520 & NGC 1241 & 13.26 & 4036 & 3 & $\mathrm{Y}$ & 0.61 & $\mathrm{~S} 2$ \\
\hline 032448.7 & -030232 & NGC 1320 & 13.67 & 2698 & 2 & $\mathrm{~N}$ & 0.32 & $\mathrm{~S} 2$ \\
\hline 032504.9 & -121807 & MCG -02-09- & 14.93 & 4400 & -2 & $\mathrm{~N}$ & 0.33 & $\mathrm{~S} 2$ \\
\hline 032525.3 & -060839 & MRK 0609 & 15.06 & 10236 & -5 & $\mathrm{~N}$ & 0.75 & S2 \\
\hline 033040.9 & -030816 & MRK 0612 & 15.10 & 6195 & 3 & $\mathrm{Y}$ & 0.62 & $\mathrm{~S} 2$ \\
\hline 033339.7 & -050522 & NGC 1358 & 13.30 & 4013 & 0 & $\mathrm{Y}$ & 0.77 & $\mathrm{~S} 2$ \\
\hline 033336.4 & -360825 & NGC 1365 & 10.34 & 1659 & 3 & $\mathrm{Y}$ & 0.71 & S1 \\
\hline 033646.4 & -355958 & NGC 1386 & 12.64 & 924 & -2 & $\mathrm{~N}$ & 0.38 & $\mathrm{~S} 2$ \\
\hline 033703.2 & -251457 & ESO 482- G 014 & 15.39 & 12889 & 1 & $\mathrm{~N}$ & 0.40 & S2 \\
\hline 034203.2 & -211450 & ESO 5 & 12.92 & 4341 & 1 & $\mathrm{~N}$ & 0.87 & $\mathrm{~S} 1$ \\
\hline 034326.5 & -314438 & GSC 702800092 & 14.90 & 9574 & 3 & $\mathrm{~N}$ & 0.64 & S2 \\
\hline 034512.5 & -393430 & GSC 756901468 & 15.48 & 12884 & -5 & $\mathrm{~N}$ & 0.96 & $\mathrm{~S} 1$ \\
\hline 035509.3 & -172810 & ESO 549- G 036 & 14.64 & 8501 & 4 & $\mathrm{Y}$ & 0.75 & $\mathrm{~S} 2$ \\
\hline 035738.2 & -191259 & NGC 1489 & 14.36 & 11421 & 3 & $\mathrm{Y}$ & 0.44 & $\mathrm{~S} 2$ \\
\hline 040225.7 & -180251 & ESO 549- G 049 & 14.36 & 7872 & 3 & $\mathrm{~N}$ & 0.75 & S2 \\
\hline 040246.1 & -210709 & ESO 549- G 050 & 14.17 & 7532 & 3 & $\mathrm{~N}$ & 0.67 & $\mathrm{~S} 2$ \\
\hline 040427.5 & -101007 & MCG -02-11-014 & 15.41 & 9267 & 2 & $\mathrm{Y}$ & 0.62 & $\mathrm{~S} 2$ \\
\hline 041349.7 & -320025 & ESO 420- G 013 & 13.52 & 3594 & 0 & $\mathrm{~N}$ & 0.90 & S2 \\
\hline 043937.3 & -370508 & GSC 704501444 & 15.22 & 12292 & 3 & $\mathrm{~N}$ & 0.48 & $\mathrm{~S} 2$ \\
\hline 044059.6 & -373411 & ESO 304- G 011 & 15.34 & 12392 & 3 & $\mathrm{Y}$ & 0.45 & $\mathrm{~S} 2$ \\
\hline 094233.3 & -034155 & NGC 2974 & 12.30 & 1908 & 1 & $\mathrm{~N}$ & 0.57 & $\mathrm{~S} 2$ \\
\hline 095056.5 & -045907 & MCG -01-25-049 & 14.46 & 6628 & 1 & $\mathrm{Y}$ & 0.42 & $\mathrm{~S} 2$ \\
\hline
\end{tabular}


Table 1-Continued

\begin{tabular}{|c|c|c|c|c|c|c|c|c|}
\hline $\begin{array}{l}\text { Coordinates } \\
\alpha\left(\begin{array}{lll}h & m s\end{array}\right)\end{array}$ & $\begin{array}{c}(\mathrm{J} 2000.0) \\
\delta\left({ }^{\circ} \prime^{\prime \prime}\right)\end{array}$ & Identification & $m_{S S R S}$ & $\begin{array}{l}\text { Velocity } \\
k m s^{-1}\end{array}$ & Type & Bar & $b / a$ & Sey \\
\hline 100200.1 & -080942 & GSC 547601170 & 15.22 & 4569 & 15 & $\mathrm{~N}$ & 0.90 & S1 \\
\hline 101803.3 & -034741 & GSC 490701728 & 15.36 & 11947 & 2 & $\mathrm{~N}$ & 1.00 & $\mathrm{~S} 2$ \\
\hline 102057.8 & -045703 & GSC 490801685 & 15.25 & 11889 & 1 & $\mathrm{Y}$ & 1.00 & $\mathrm{~S} 2$ \\
\hline 102550.2 & -112607 & MCG -02-27-003 & 15.00 & 11858 & 0 & $\mathrm{~N}$ & 1.00 & $\mathrm{~S} 2$ \\
\hline 102651.9 & -032752 & IC 0614 & 15.39 & 10194 & 0 & $\mathrm{~N}$ & 0.71 & $\mathrm{~S} 2$ \\
\hline 103527.3 & -140707 & MCG -02-27-009 & 14.33 & 4577 & 2 & $\mathrm{~N}$ & 0.26 & $\mathrm{~S} 2$ \\
\hline 103820.6 & -100704 & GSC 549500478 & 15.41 & 8710 & 2 & $\mathrm{Y}$ & 0.50 & S1 \\
\hline 103946.3 & -052900 & MCG -01-27-031 & 14.07 & 6194 & 1 & $\mathrm{Y}$ & 0.75 & S1 \\
\hline 104218.7 & -173807 & MCG -03-27-026 & 14.80 & 6191 & -2 & $\mathrm{~N}$ & 0.29 & S2 \\
\hline 105753.6 & -045418 & IC 0657 & 15.44 & 9697 & 1 & $\mathrm{Y}$ & 0.42 & $\mathrm{~S} 2$ \\
\hline 110045.4 & -063443 & GSC 492701023 & 15.36 & 8997 & 3 & $\mathrm{~N}$ & 0.24 & $\mathrm{~S} 2$ \\
\hline 112332.2 & -083930 & NGC 3660 & 13.06 & 3678 & 4 & $\mathrm{Y}$ & 0.87 & $\mathrm{~S} 2$ \\
\hline 112456.3 & -034841 & GSC 492601061 & 15.04 & 6788 & 2 & $\mathrm{~N}$ & 0.40 & $\mathrm{~S} 2$ \\
\hline 112502.1 & -050407 & MCG -01-29-018 & 14.53 & 8518 & 2 & $\mathrm{~N}$ & 0.90 & $\mathrm{~S} 2$ \\
\hline 113423.3 & -094007 & MCG -01-30-005 & 15.12 & 6419 & -3 & $\mathrm{~N}$ & 1.00 & $\mathrm{~S} 2$ \\
\hline 114318.6 & -125242 & NGC 3831 & 13.60 & 5254 & 1 & $\mathrm{~N}$ & 0.22 & $\mathrm{~S} 2$ \\
\hline 114511.6 & -091851 & NGC 3858 & 13.94 & 5729 & 0 & $\mathrm{Y}$ & 0.67 & S2 \\
\hline 114540.5 & -182716 & GSC 609600769 & 14.70 & 9877 & 2 & $\mathrm{~N}$ & 0.55 & S1 \\
\hline 115238.2 & -051207 & MCG -01-30-041 & 14.70 & 5749 & 0 & $\mathrm{Y}$ & 0.50 & S2 \\
\hline 120043.3 & -205001 & GSC 610000370 & 15.46 & 18639 & 15 & $\mathrm{~N}$ & 0.76 & S1 \\
\hline 121660.0 & -261236 & ESO 505-IG 031 & 15.49 & 11840 & 15 & $\mathrm{Y}$ & 0.88 & $\mathrm{~S} 2$ \\
\hline 123939.4 & -052039 & NGC 4593 & 12.21 & 2698 & 2 & $\mathrm{Y}$ & 0.83 & S1 \\
\hline 123959.4 & -113723 & NGC 4594 & 9.50 & 994 & 0 & $\mathrm{~N}$ & 0.62 & $\mathrm{~S} 2$ \\
\hline 124225.3 & -065816 & NGC 4628 & 14.50 & 2828 & 1 & $\mathrm{~N}$ & 0.23 & $\mathrm{~S} 2$ \\
\hline 125132.4 & -141317 & MCG -02-33-030 & 14.23 & 4298 & 1 & $\mathrm{~N}$ & 0.43 & S1 \\
\hline $12 \quad 52 \quad 12.4$ & -132454 & NGC 4748 & 14.27 & 4426 & 15 & $\mathrm{~N}$ & 0.86 & S1 \\
\hline 125236.4 & -215439 & ESO 575-IG 016 NED02 & 14.87 & 6865 & 15 & $\mathrm{~N}$ & 0.70 & $\mathrm{~S} 2$ \\
\hline 125636.3 & -064903 & NGC 4813 & 14.15 & 1373 & 1 & $\mathrm{~N}$ & 0.40 & $\mathrm{~S} 2$ \\
\hline 130052.9 & -132659 & NGC 4897 & 13.23 & 2546 & 3 & $\mathrm{Y}$ & 0.80 & $\mathrm{~S} 2$ \\
\hline 130414.3 & -102023 & NGC 4939 & 11.99 & 3117 & 3 & $\mathrm{~N}$ & 0.38 & $\mathrm{~S} 2$ \\
\hline 130413.1 & -053306 & NGC 4941 & 12.43 & 1108 & 1 & $\mathrm{~N}$ & 0.48 & $\mathrm{~S} 2$ \\
\hline 130656.5 & -235501 & IC 4180 & 13.88 & 2972 & 0 & $\mathrm{Y}$ & 0.77 & $\mathrm{~S} 2$ \\
\hline 130705.9 & -234039 & NGC 4968 & 13.92 & 2930 & 0 & $\mathrm{~N}$ & 0.47 & $\mathrm{~S} 2$ \\
\hline 131017.3 & -072707 & MCG -01-34-008 & 14.54 & 6713 & 3 & $\mathrm{~N}$ & 0.86 & $\mathrm{~S} 2$ \\
\hline 131703.4 & -021541 & IC 4218 & 14.90 & 5808 & 1 & $\mathrm{~N}$ & 0.27 & S1 \\
\hline 131931.7 & -123926 & NGC 5077 & 12.41 & 2817 & -5 & $\mathrm{~N}$ & 0.50 & $\mathrm{~S} 2$ \\
\hline 132006.4 & -170706 & MCG -03-34-049 & 15.14 & 6713 & 0 & $\mathrm{~N}$ & 0.83 & S1 \\
\hline
\end{tabular}


Table 1-Continued

\begin{tabular}{|c|c|c|c|c|c|c|c|c|}
\hline $\begin{array}{l}\text { Coordinates } \\
\alpha\left(\begin{array}{lll}h & m s\end{array}\right)\end{array}$ & $\begin{array}{c}(\mathrm{J} 2000.0) \\
\delta\left({ }^{\circ} \prime \prime \prime\right)\end{array}$ & Identification & $m_{S S R S}$ & $\begin{array}{c}\text { Velocity } \\
k m s^{-1}\end{array}$ & Type & Bar & $b / a$ & Sey \\
\hline 132224.5 & -164307 & MCG -03-34-064 & 14.64 & 6393 & -2 & $\mathrm{~N}$ & 0.80 & $\mathrm{~S} 2$ \\
\hline 132435.2 & -194511 & GSC 612800106 & 14.97 & 5284 & 3 & $\mathrm{~N}$ & 0.77 & $\mathrm{~S} 2$ \\
\hline 132712.7 & -245141 & GSC 671700254 & 15.42 & 12131 & 2 & $\mathrm{~N}$ & 0.79 & S1 \\
\hline 133041.7 & -213947 & GSC 613300078 & 15.49 & 7292 & 0 & $\mathrm{Y}$ & 0.73 & $\mathrm{~S} 2$ \\
\hline 133113.9 & -252410 & ESO 509- G 038 & 14.74 & 7786 & 15 & $\mathrm{~N}$ & 0.42 & S1 \\
\hline 133239.1 & -102853 & MCG -02-35-001 & 14.82 & 6638 & 2 & $\mathrm{Y}$ & 0.67 & S1 \\
\hline 133308.3 & -233237 & GSC 671400714 & 14.50 & 10342 & 3 & $\mathrm{~N}$ & 0.81 & $\mathrm{~S} 2$ \\
\hline 133439.6 & -232648 & ESO 509-IG 066 & 14.70 & 10031 & 15 & $\mathrm{~N}$ & 0.77 & $\mathrm{~S} 2$ \\
\hline 133735.1 & -211258 & GSC 613400646 & 15.26 & 15703 & 3 & $\mathrm{~N}$ & 0.49 & $\mathrm{~S} 2$ \\
\hline 133750.0 & -235941 & ESO 509-IG 083 NED01 & 14.70 & 9044 & 15 & $\mathrm{~N}$ & 0.63 & $\mathrm{~S} 2$ \\
\hline 134019.7 & -235129 & NGC 5260 & 13.42 & 6539 & 2 & $\mathrm{Y}$ & 0.88 & $\mathrm{~S} 2$ \\
\hline 134620.1 & -032542 & MCG +00-35-020 NED02 & 15.02 & 6948 & 2 & $\mathrm{~N}$ & 0.38 & S2 \\
\hline 135859.7 & -200244 & GSC 614400913 & 15.50 & 11797 & -5 & $\mathrm{~N}$ & 1.00 & $\mathrm{~S} 2$ \\
\hline 140326.1 & -060151 & NGC 5427 & 12.50 & 2618 & 3 & $\mathrm{~N}$ & 1.00 & $\mathrm{~S} 2$ \\
\hline 141314.9 & -031227 & NGC 5506 & 13.37 & 1753 & 3 & $\mathrm{~N}$ & 0.57 & $\mathrm{~S} 2$ \\
\hline 142612.3 & -115407 & MCG -02-37-004 & 15.48 & 12480 & 3 & $\mathrm{Y}$ & 0.80 & $\mathrm{~S} 2$ \\
\hline 143343.5 & -143711 & NGC 5664 & 14.52 & 4537 & 2 & $\mathrm{~N}$ & 0.42 & $\mathrm{~S} 2$ \\
\hline 144223.9 & -171510 & NGC & 12.81 & 2793 & 4 & $\mathrm{Y}$ & 0.58 & $\mathrm{~S} 2$ \\
\hline 145924.8 & -164136 & NGC 5793 & 14.17 & 3522 & 2 & $\mathrm{~N}$ & 0.35 & $\mathrm{~S} 2$ \\
\hline 153320.7 & -084207 & MCG $-01-4$ & 14.64 & 6888 & 4 & $\mathrm{Y}$ & 0.38 & $\mathrm{~S} 2$ \\
\hline 210759.9 & -295010 & GSC 693600748 & 15.10 & 5750 & 1 & $\mathrm{~N}$ & 0.90 & $\mathrm{~S} 2$ \\
\hline 212702.8 & -225932 & ESO 530- G 047 & 14.51 & 9761 & 2 & $\mathrm{~N}$ & 0.59 & $\mathrm{~S} 2$ \\
\hline 214819.5 & -345706 & NGC 7130 & 13.33 & 4829 & 15 & $\mathrm{~N}$ & 0.94 & $\mathrm{~S} 2$ \\
\hline 215656.6 & -113931 & GSC 579701005 & 15.37 & 17650 & 1 & $\mathrm{~N}$ & 0.54 & S1 \\
\hline 220021.6 & -130849 & IC 1417 & 14 & 5446 & 3 & $\mathrm{~N}$ & 0.29 & $\mathrm{~S} 2$ \\
\hline 220117.0 & -374607 & MCG -06-48-013 & 14.82 & 10005 & -2 & $\mathrm{~N}$ & 0.75 & $\mathrm{~S} 2$ \\
\hline 220201.7 & -315218 & NGC 7172 & 12.95 & 2593 & 1 & $\mathrm{~N}$ & 0.52 & $\mathrm{~S} 2$ \\
\hline 220828.3 & -340623 & ESO 404- G & 14.33 & 4431 & 4 & $\mathrm{~N}$ & 0.20 & $\mathrm{~S} 2$ \\
\hline 220907.7 & -274834 & NGC 7214 & 13.05 & 6832 & 4 & $\mathrm{~N}$ & 0.74 & $\mathrm{~S} 1$ \\
\hline 221442.0 & -384823 & ESO 344- G 016 & 15.08 & 11906 & 2 & $\mathrm{Y}$ & 1.00 & S1 \\
\hline 223449.8 & -254037 & ESO 533- G 050 & 14.40 & 7928 & 0 & $\mathrm{Y}$ & 0.89 & $\mathrm{~S} 2$ \\
\hline 223545.9 & -260301 & NGC 7314 & 11.88 & 1430 & 4 & $\mathrm{~N}$ & 0.42 & $\mathrm{~S} 2$ \\
\hline 223646.5 & -123243 & MCG -02-57-023 & 14.82 & 7169 & 4 & $\mathrm{~N}$ & 0.30 & S1 \\
\hline 223655.9 & -221315 & ESO 602- G 031 & 14.88 & 10101 & 3 & $\mathrm{~N}$ & 0.50 & $\mathrm{~S} 1$ \\
\hline 224747.6 & -114859 & NGC 7378 & 13.64 & 2580 & 2 & $\mathrm{Y}$ & 0.64 & $\mathrm{~S} 2$ \\
\hline 224937.1 & -191607 & MCG -03-58-007 & 14.84 & 9488 & 3 & $\mathrm{Y}$ & 0.80 & $\mathrm{~S} 2$ \\
\hline 225415.7 & -370459 & ESO 406- G 018 & 15.29 & 17215 & 15 & $\mathrm{~N}$ & 0.54 & S2 \\
\hline
\end{tabular}


Table 1 - Continued

\begin{tabular}{|c|c|c|c|c|c|c|c|c|}
\hline $\begin{array}{l}\text { Coordinates } \\
\alpha\left(\begin{array}{lll}h & m & s\end{array}\right)\end{array}$ & $\begin{array}{c}(\mathrm{J} 2000.0) \\
\delta\left({ }^{\circ} \prime \prime \prime\right)\end{array}$ & Identification & $m_{S S R S}$ & $\begin{array}{c}\text { Velocity } \\
k m s^{-1}\end{array}$ & Type & Bar & $b / a$ & Sey \\
\hline 225501.0 & -393941 & NGC 7410 & 11.61 & 1751 & 1 & $\mathrm{Y}$ & 0.29 & $\mathrm{~S} 2$ \\
\hline 225901.4 & -253142 & ESO 535- G 001 & 13.84 & 9015 & 2 & $\mathrm{~N}$ & 0.94 & S2 \\
\hline 230047.8 & -125507 & NGC 7450 & 14.00 & 3134 & 1 & $\mathrm{Y}$ & 1.00 & $\mathrm{~S} 1$ \\
\hline 230311.1 & -085921 & IC 1464 & 14.90 & 7247 & 15 & $\mathrm{~N}$ & 0.67 & $\mathrm{~S} 2$ \\
\hline 230443.5 & -084109 & MCG -02-58-022 & 15.00 & 14185 & 1 & $\mathrm{~N}$ & 1.00 & S1 \\
\hline 231637.4 & -021950 & NGC 7566 & 14.27 & 7972 & 1 & $\mathrm{Y}$ & 0.54 & S2 \\
\hline 231822.5 & -042459 & NGC 7592 & 14.50 & 7328 & 15 & $\mathrm{~N}$ & 0.80 & $\mathrm{~S} 2$ \\
\hline 232524.2 & -382651 & GSC 8013012 & 14.89 & 10761 & 5 & $\mathrm{~N}$ & 1.00 & $\mathrm{~S} 1$ \\
\hline 233032.3 & -022745 & MCG -01-59-027 & 15.14 & 10006 & 0 & $\mathrm{Y}$ & 0.68 & S1 \\
\hline 233047.7 & -132908 & IC 1495 & 14.14 & 6391 & 2 & $\mathrm{Y}$ & 0.77 & $\mathrm{~S} 2$ \\
\hline 234205.2 & -391300 & GSC 801400236 & 15.42 & 12828 & 3 & $\mathrm{~N}$ & 0.71 & S1 \\
\hline 235319.8 & -300903 & ESO 471-IG 037 NED02 & 15.24 & 14660 & 15 & $\mathrm{~N}$ & 0.65 & $\mathrm{~S} 2$ \\
\hline 235728.0 & -302741 & MCG -05-01-013 & 14.97 & 9080 & 3 & $\mathrm{~N}$ & 0.56 & S1 \\
\hline 235910.7 & -040737 & IC 1524 & 13.94 & 5662 & 2 & $\mathrm{Y}$ & 0.62 & S1 \\
\hline
\end{tabular}

Table 2. SSRS2 and Seyfert galaxies versus environment assignement

\begin{tabular}{ccccc}
\hline \hline & Groups & Triplets & Binaries & Isolated \\
\hline Seyferts & $21 \%$ & $7 \%$ & $28 \%$ & $44 \%$ \\
SSRS2 & $24 \%$ & $8 \%$ & $18 \%$ & $50 \%$ \\
\hline
\end{tabular}




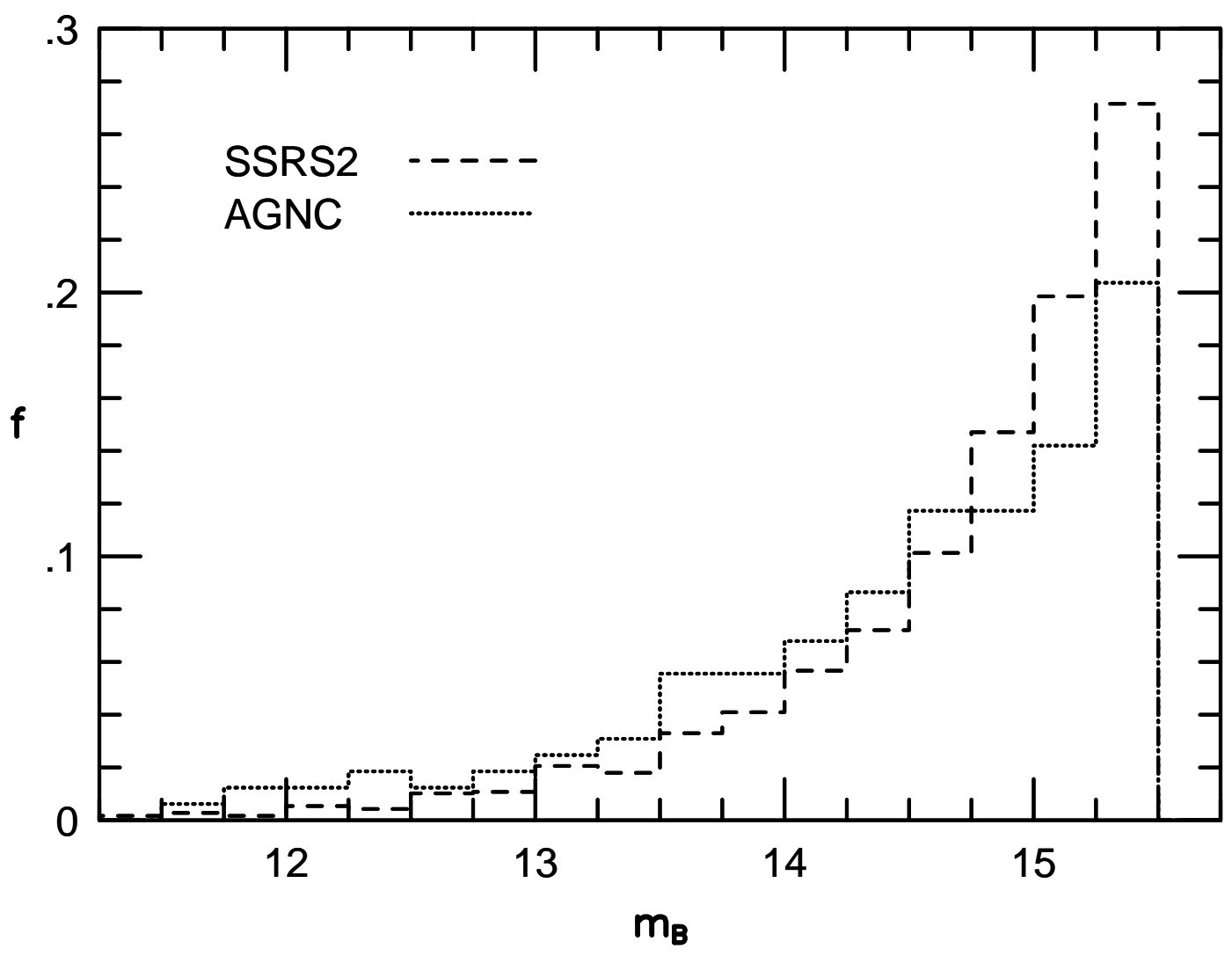

Fig. 1.- Apparent magnitude distribution of galaxies in the SSRS2 (dashed line) and AGNC (solid line) samples. 


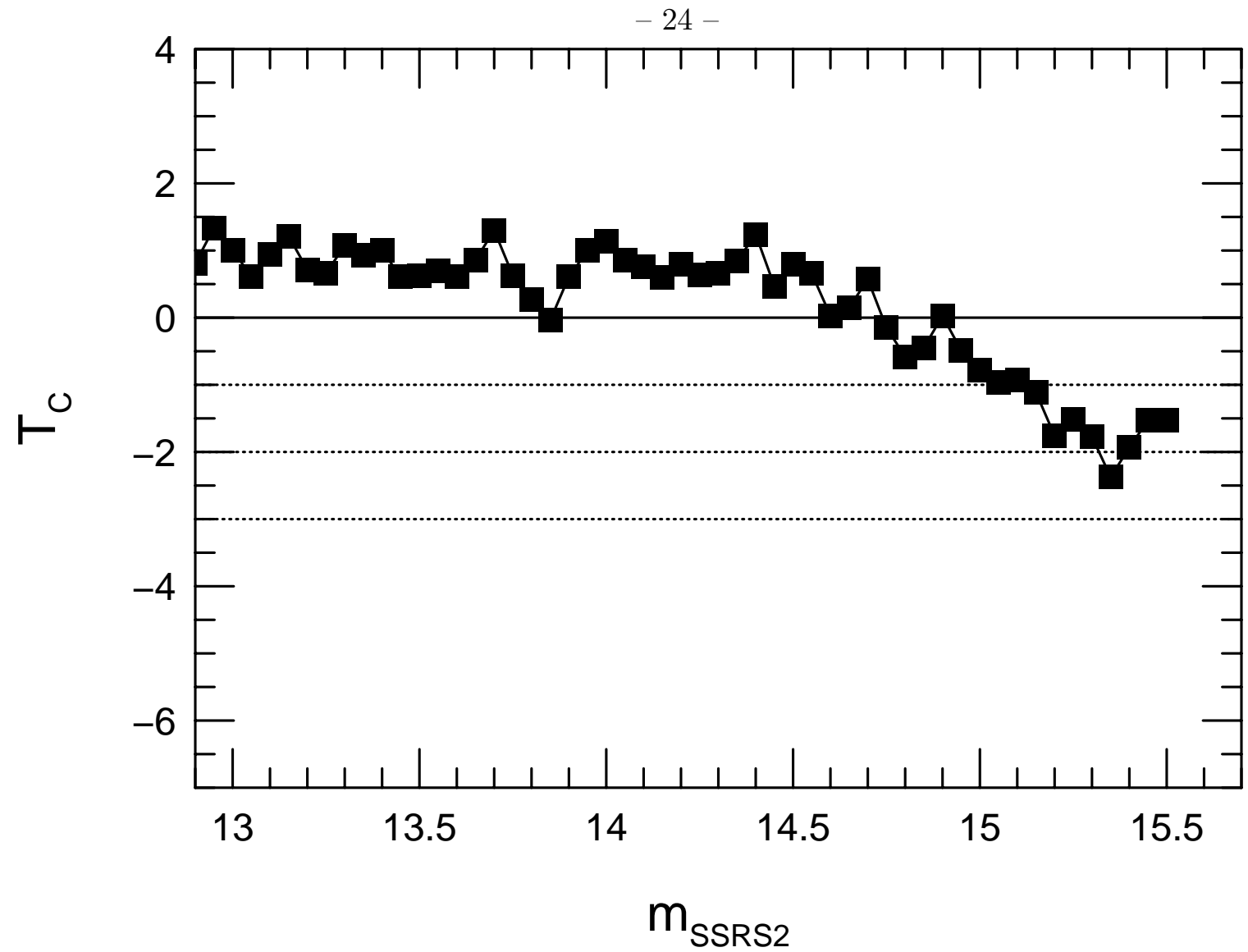

Fig. 2.- Completeness test of Rauzy (2001) which shows the distribution of SSRS2 AGNs at different limiting magnitudes (represented as squares) and the different confidence levels where the catalog completeness may be rejected in number of standard deviations $(0 \%, 68 \%, 90 \%$ and $95 \%)$, represented by the horizontal lines. The sample completeness cannot be rejected at the $95 \%$ confidence level at the limiting apparent magnitude. 


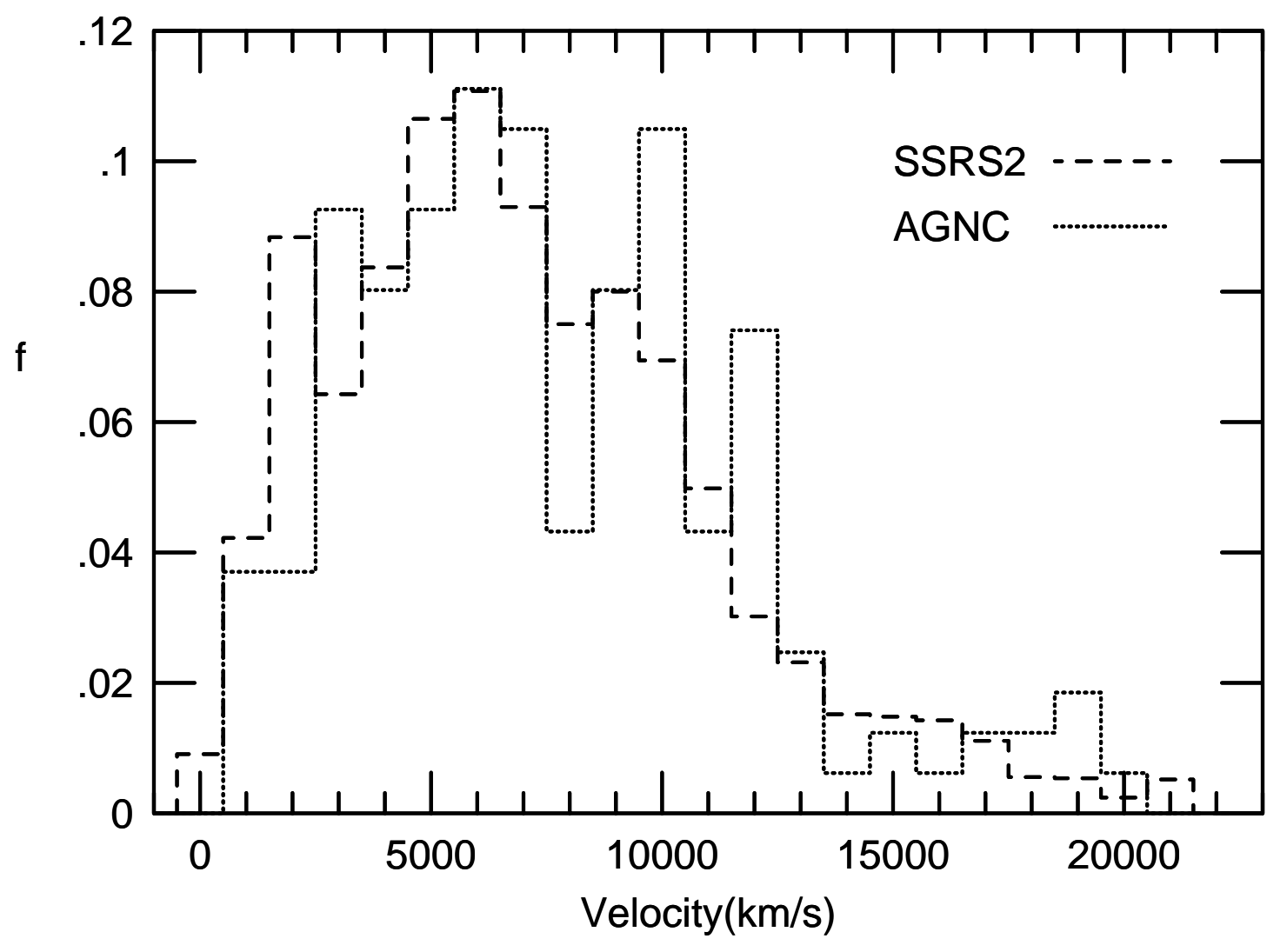

Fig. 3.- Radial velocity distribution of galaxies in the AGNC (solid line) and SSRS2 (dashed line). 


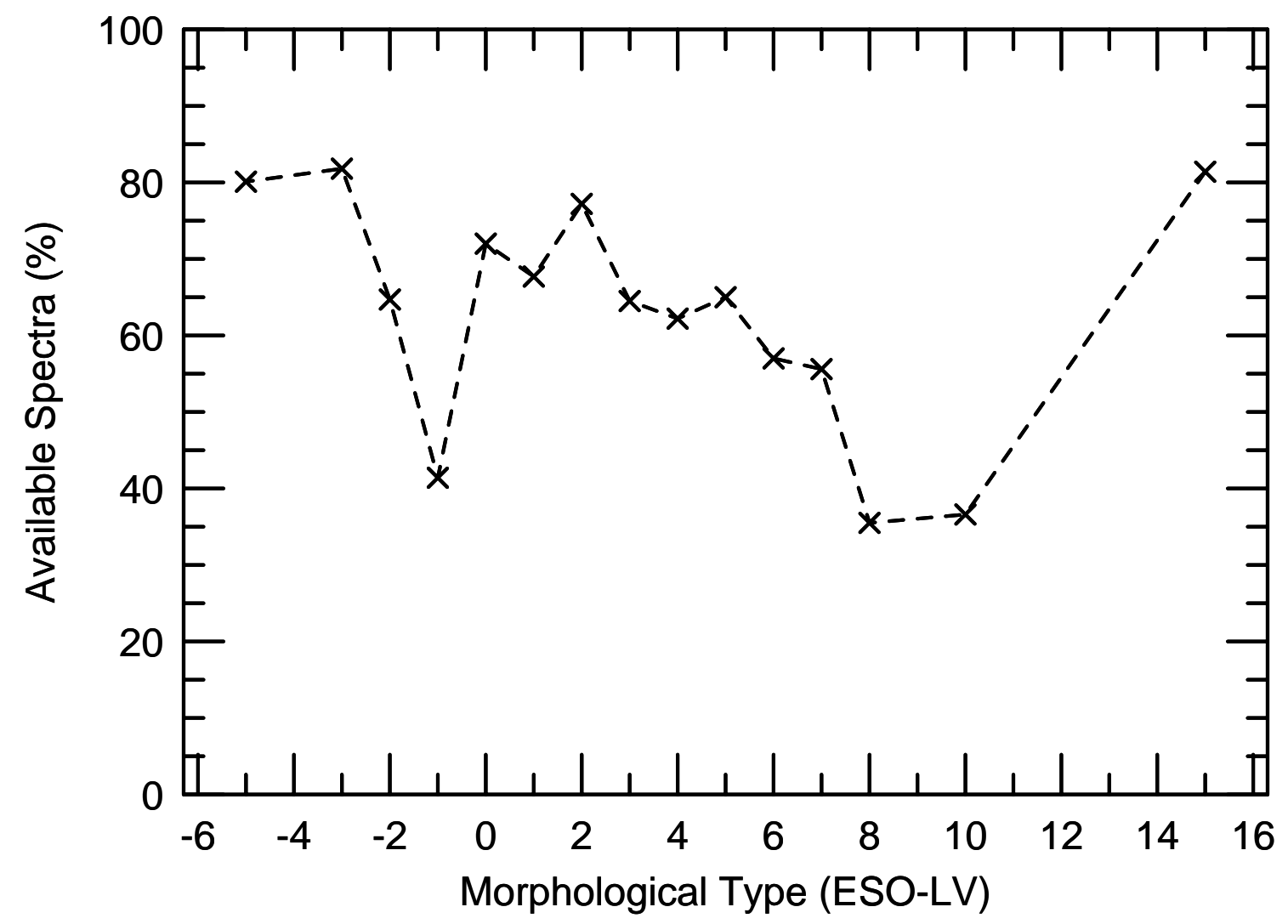

Fig. 4.- Percentage of SSRS2 galaxies which have spectra available in our database as a function of galaxy morphology. The numbers correspond to the following classification: $\mathrm{E}=-5, \mathrm{E} / \mathrm{S} 0=-3$, $\mathrm{S} 0=-2, \mathrm{~S} 0 \mathrm{a}=0, \mathrm{Sa}=1, \mathrm{Sab}=2, \mathrm{Sb}=3, \mathrm{Sbc}=4, \mathrm{~S} \ldots=5, \mathrm{Sc}=6, \mathrm{Sc} / \mathrm{Irr}=7, \mathrm{Sd}=8, \mathrm{Irr}=10$, merger $=15$. 


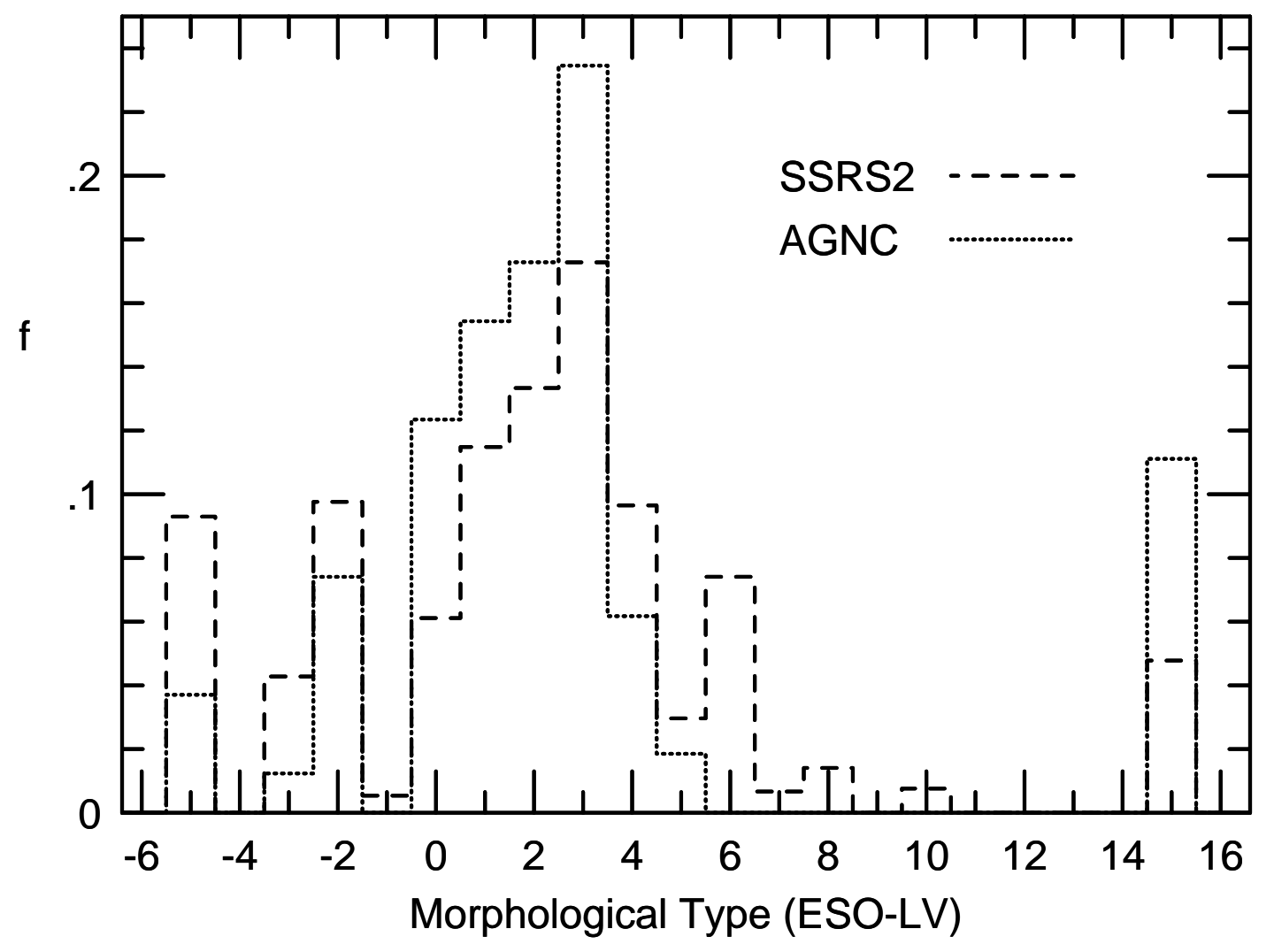

Fig. 5.- Fraction of galaxies in the AGNC (solid line) and SSRS2 (dashed line) as a function of morphological type distribution. The coding used for the morphological types is the same as in Fig. 4. 


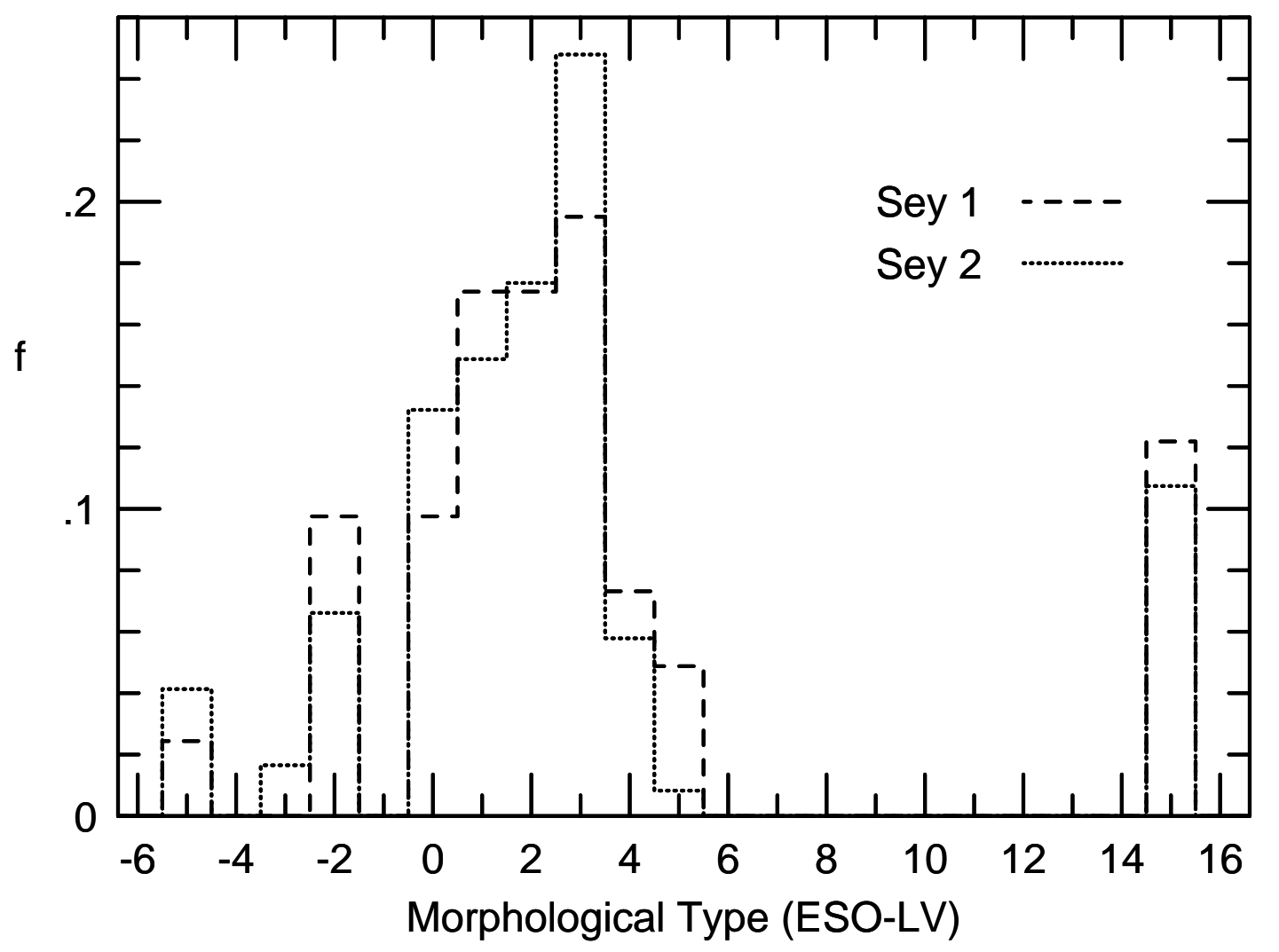

Fig. 6.- Morphological distribution of Seyfert 1 and 2. The numbers for morphological types are the same as in Fig 4. 

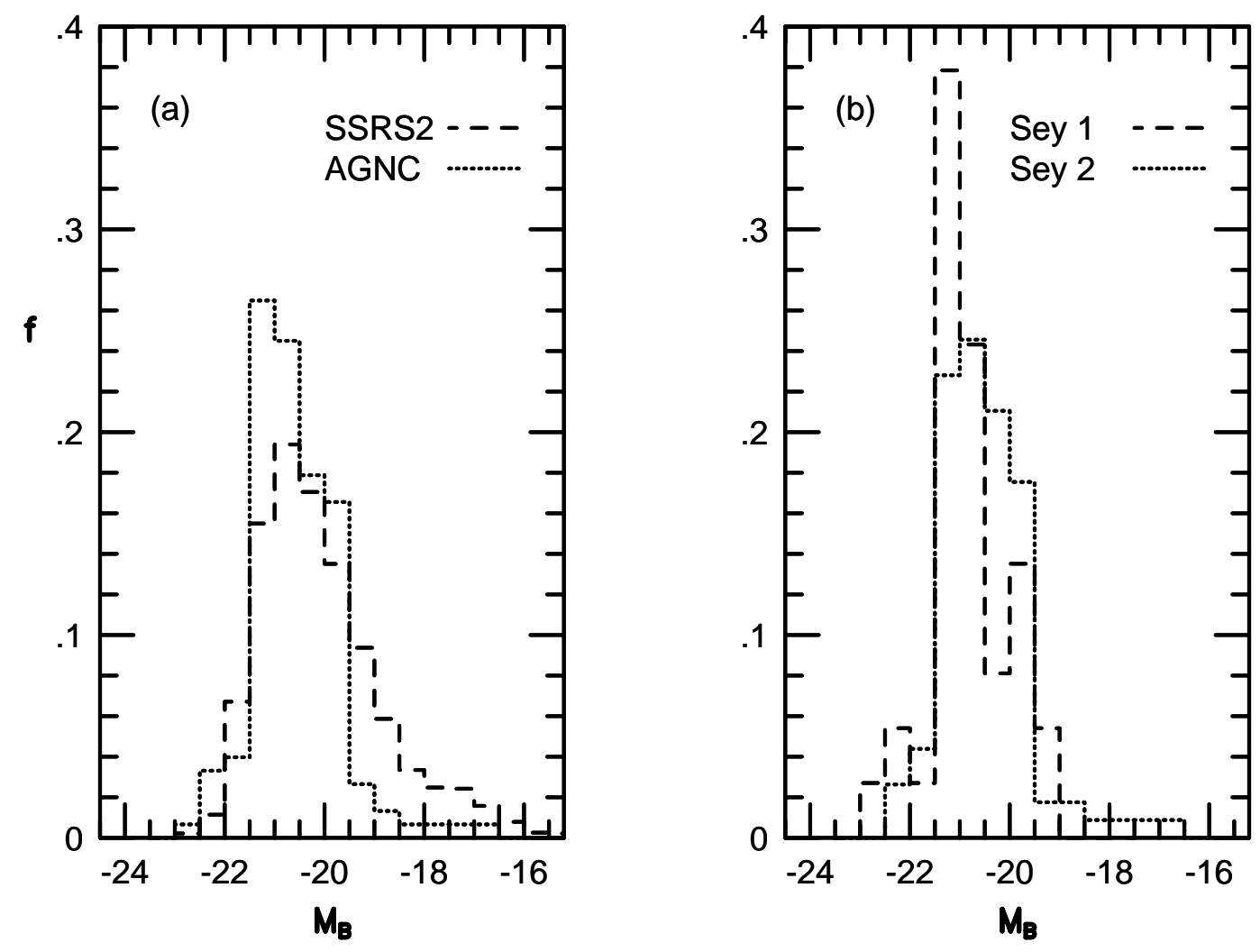

Fig. 7. - Fraction of galaxies as a function of absolute magnitude for the AGNC (solid line) and SSRS2 (dashed line) in panel (a), while panel (b) shows the same distribution for the AGNC sample divided into Seyfert 2 (solid line) and Seyfert 1 (dashed line) galaxies. 

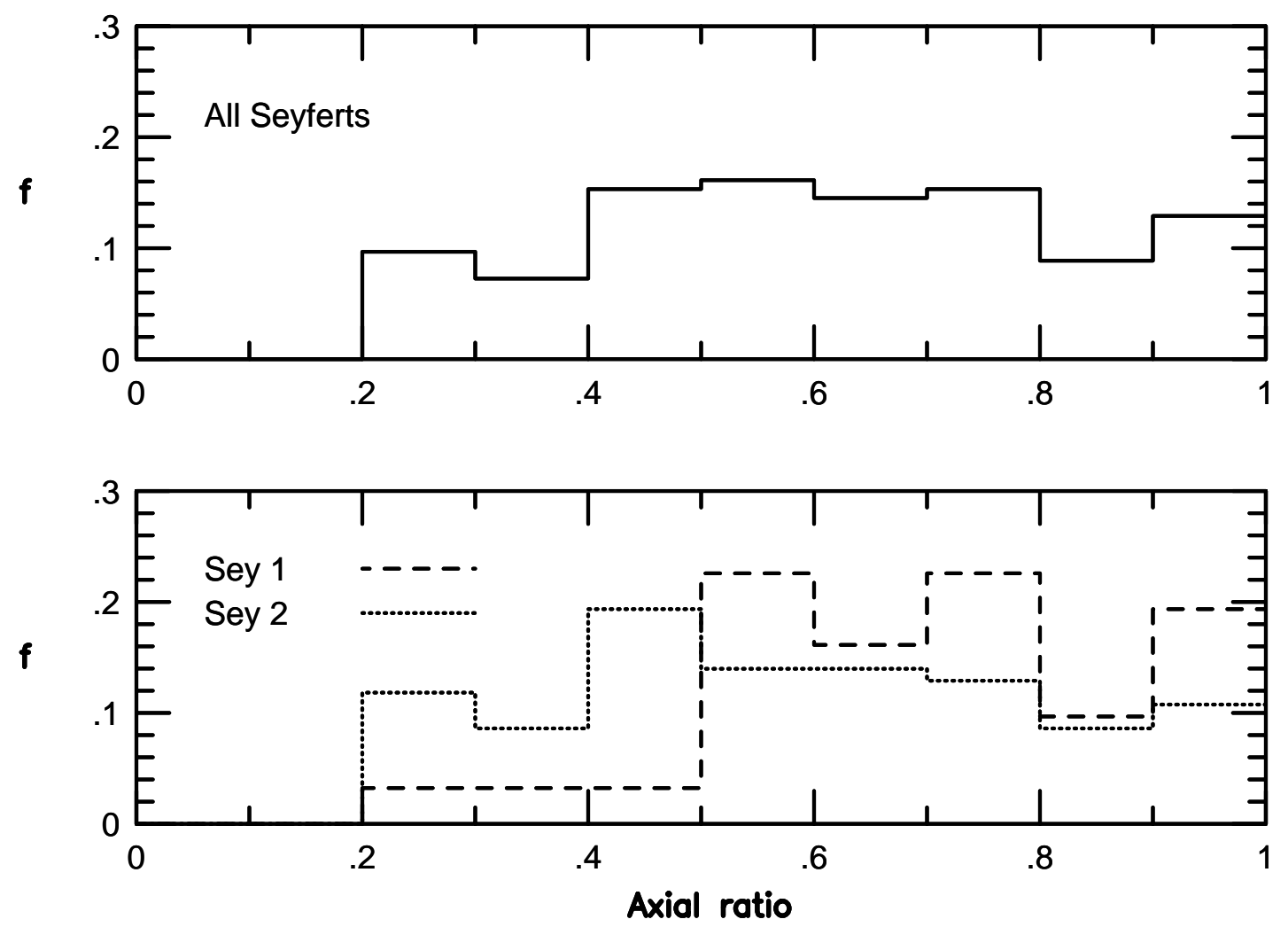

Fig. 8.- Distribution of the apparent axial ratios of AGNC hosts (panel a) and for Seyferts 1 and 2 (panel b). 


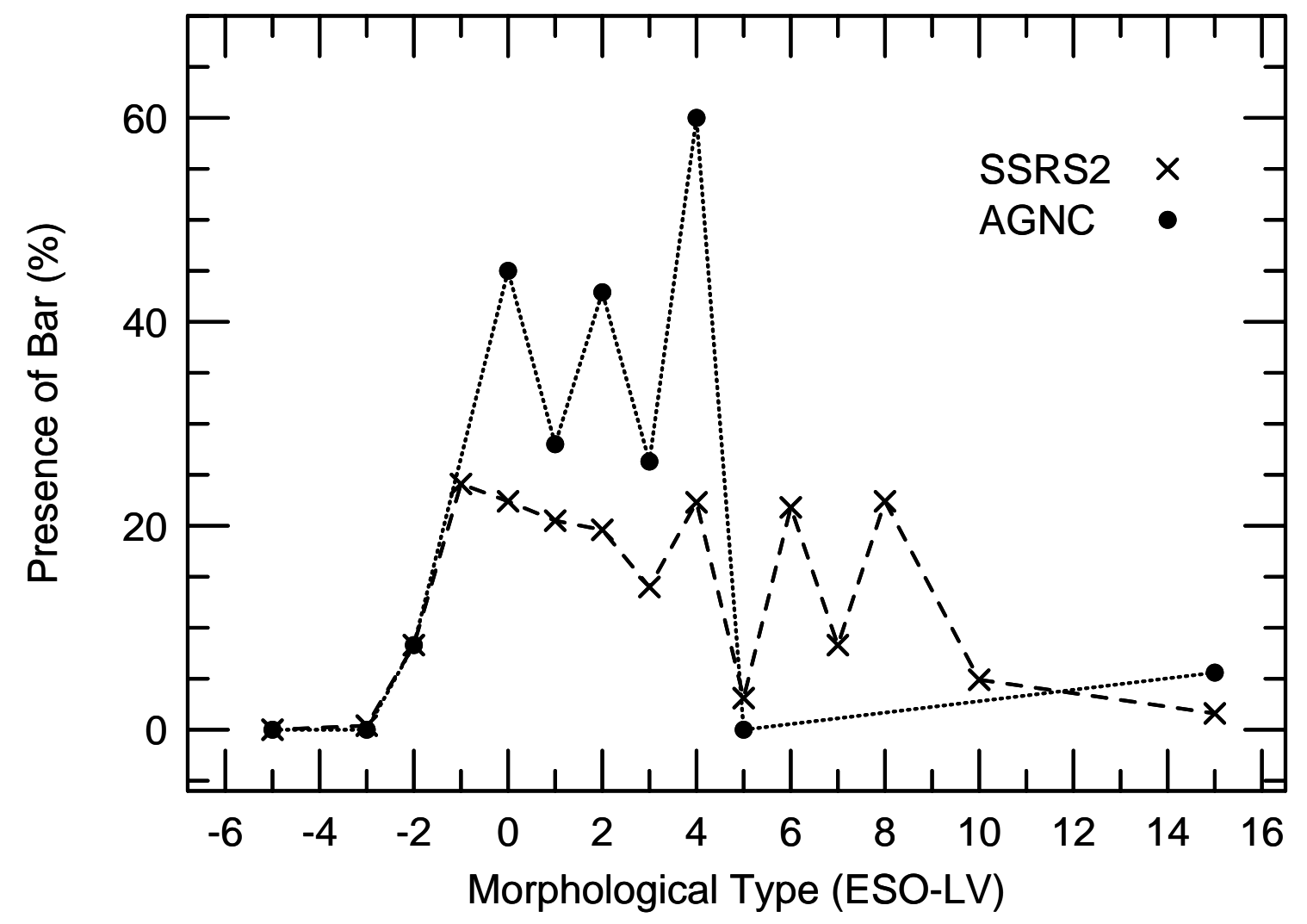

Fig. 9.- Percentage of galaxies presenting bars in the AGNC (solid line) and SSRS2 (dashed line) as a function of morpholgical type, using the same coding as in Fig. 4. 


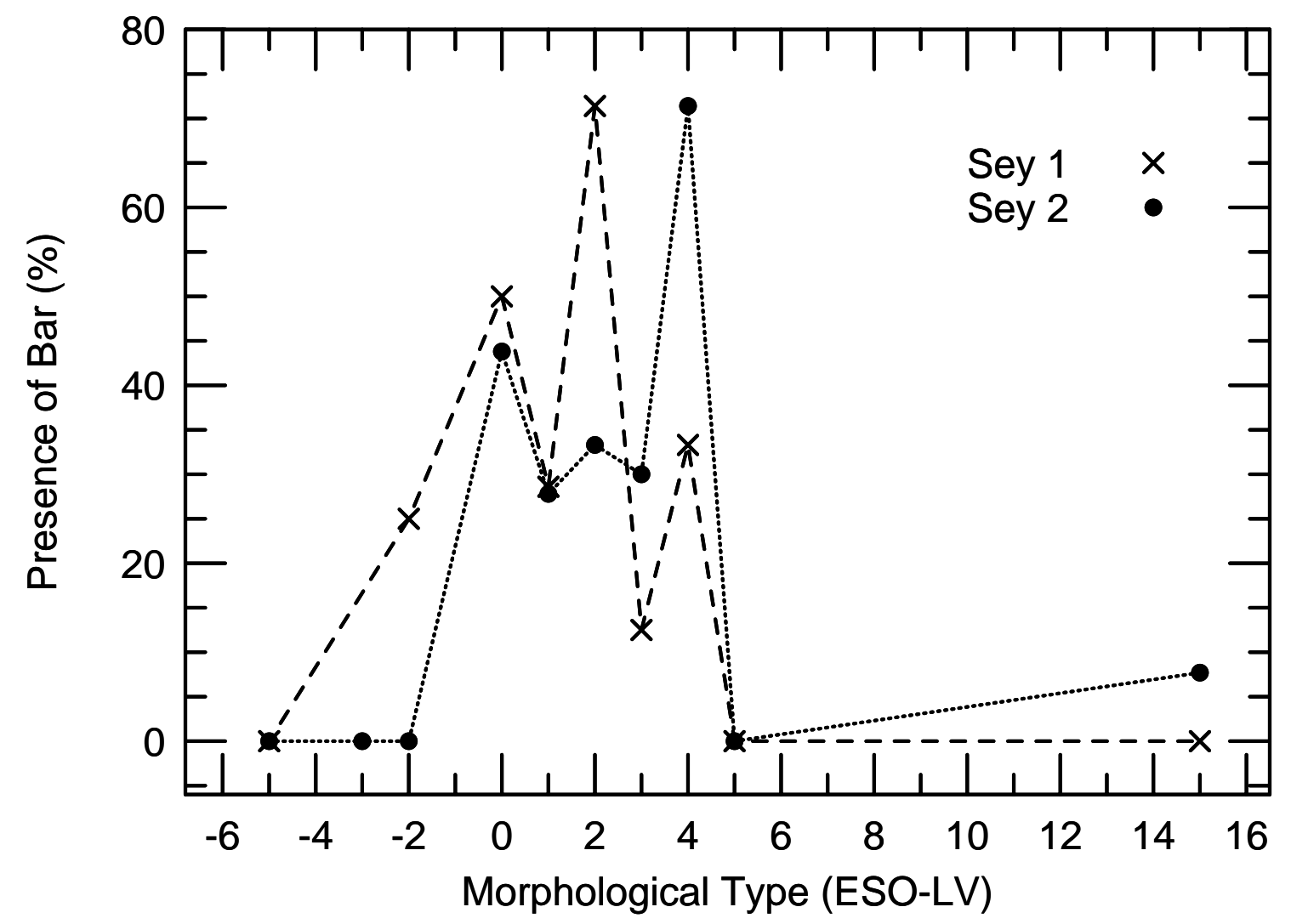

Fig. 10.- Percentage of bars of Seyferts 1 and 2. The numbers for morphological types are the same as in Fig. 4. 


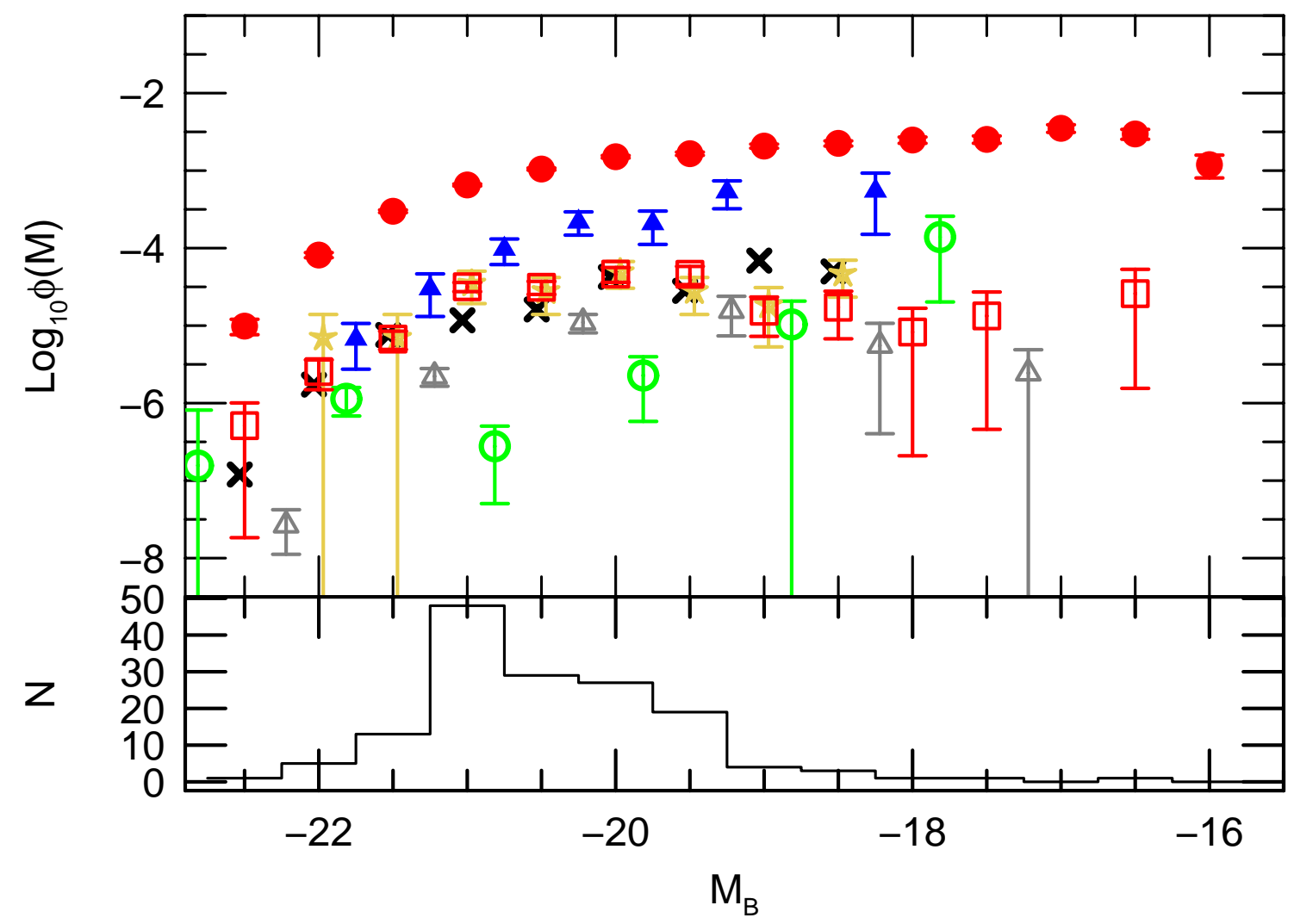

Fig. 11.- AGN Luminosity function for several low-redshift samples of galaxies. The nonparametric stepwise maximum likelihood luminosity function is shown as filled squares for the AGNC and filled circles for the SSRS2 parent sample. Also shown are previous determinations of the luminosity function: Huchra \& Burg (1992) - crosses; Köhler et al. (1997) - open circles; Londish et al. (2000) - open triangles; Ulvestad \& Ho (2001) - solid triangles; and Gronwall, Sarajedini \& Salzer (2001) - stars. 


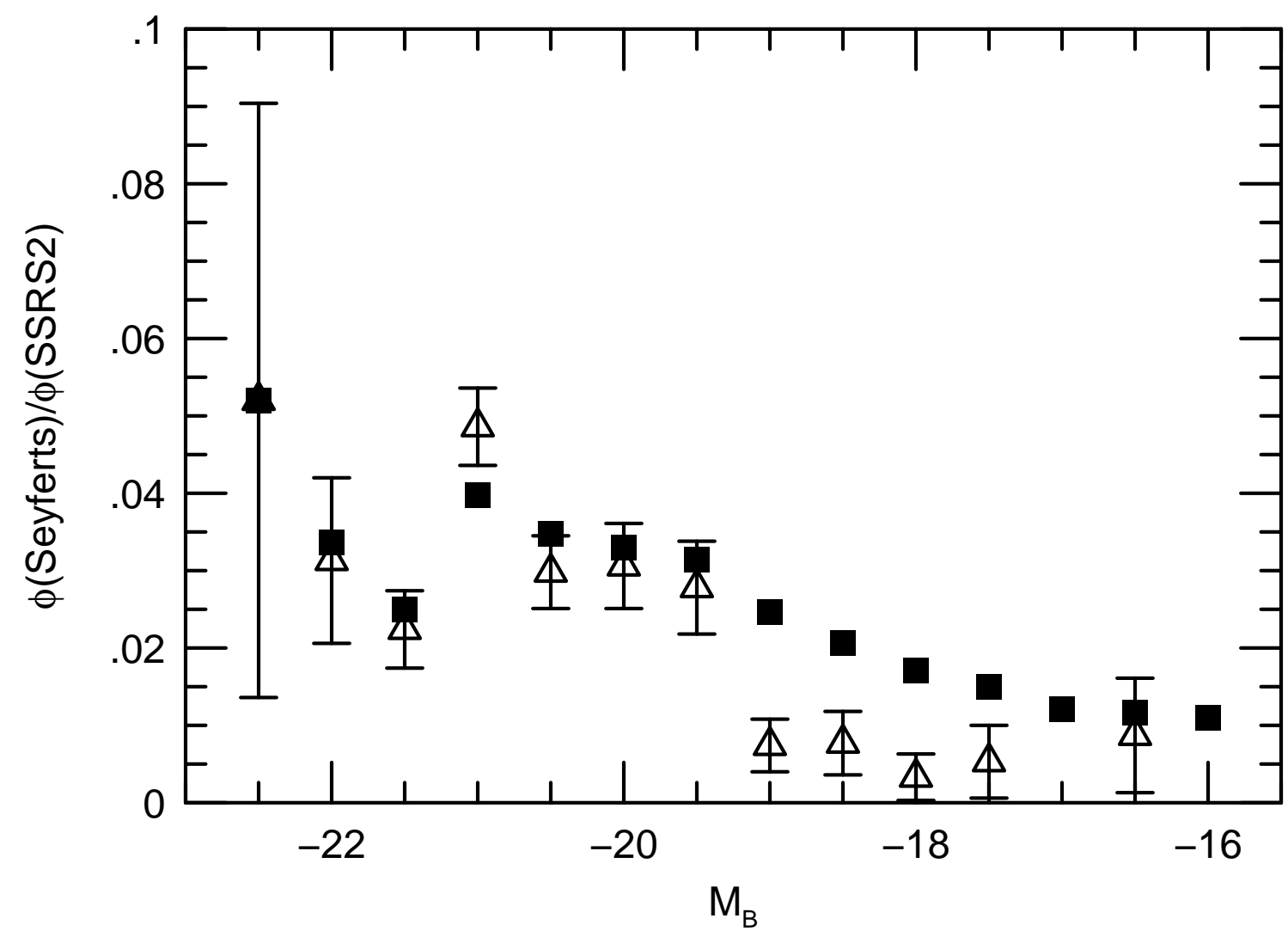

Fig. 12. - Ratio between the number densities per 0.5 magnitude bin of AGN hosts and the parent sample. The open triangles (with associated error bars) show the ratio considering only the galaxies within a given bin. The solid circles show the cumulative ratio where galaxies in a given magnitude bin and brighter are added. The figure suggests there is a trend of AGN hosts being commoner in higher luminosity galaxies. 


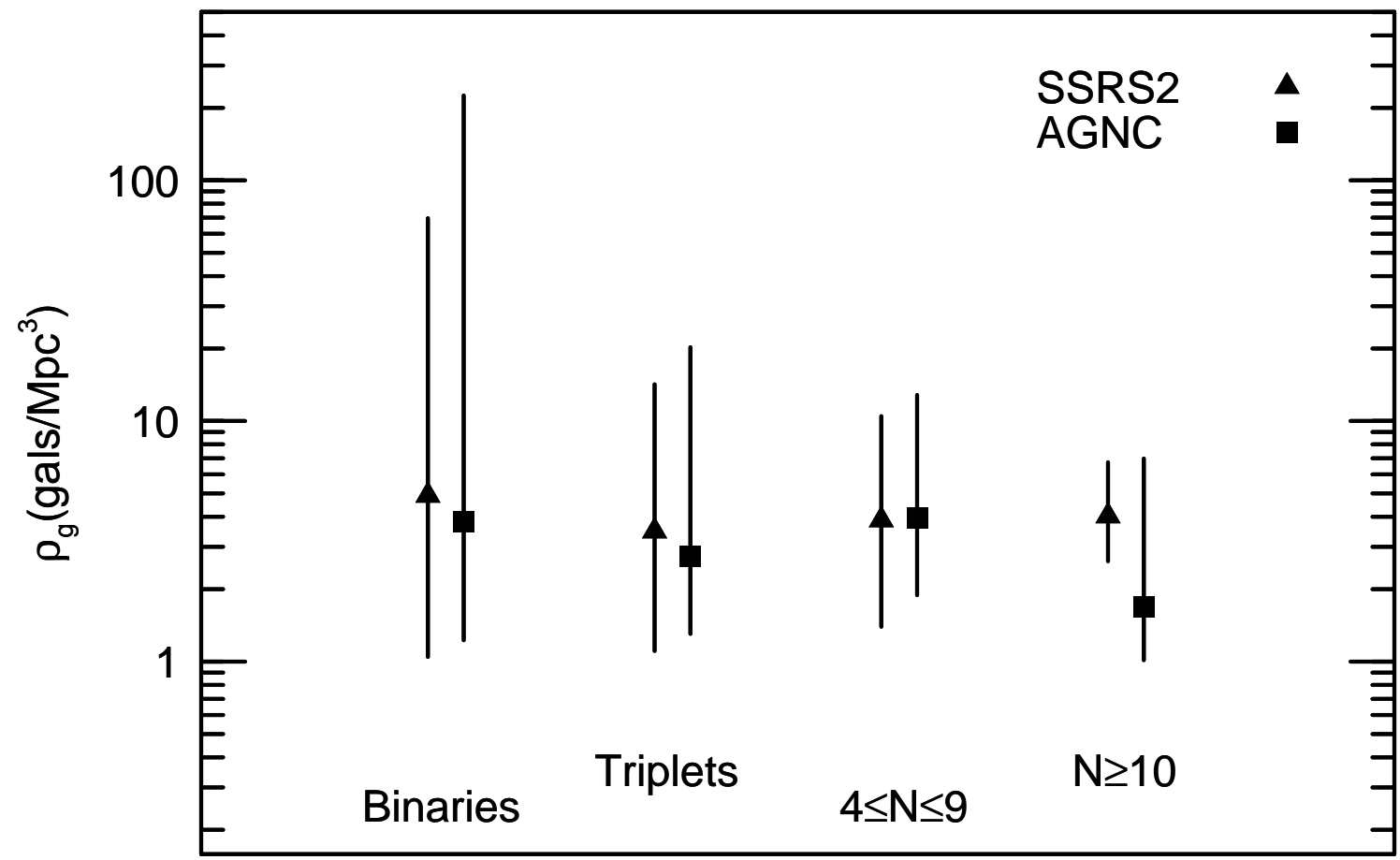

\section{Multiplicity}

Fig. 13.- Group density, $\rho_{g}$, according to multiplicity intervals. The points represent median values, while the bars refer to the upper and lower quartiles of the distributions. 